\title{
Synthetic superfluid chemistry with vortex-trapped quantum impurities
}

\author{
Matthew Edmonds $\odot,{ }^{1}$ Minoru Eto, ${ }^{2}$ and Muneto Nitta ${ }^{1}$ \\ ${ }^{1}$ Department of Physics \& Research and Education Center for Natural Sciences, Keio University, Hiyoshi 4-1-1, \\ Yokohama, Kanagawa 223-8521, Japan \\ ${ }^{2}$ Department of Physics, Yamagata University, Kojirakawa-machi 1-4-12, Yamagata, Yamagata 990-8560, Japan
}

(Received 4 November 2020; revised 2 March 2021; accepted 17 March 2021; published 3 May 2021)

\begin{abstract}
We explore the effect of using two-dimensional matter-wave vortices to confine an ensemble of bosonic quantum impurities. This is modeled theoretically using a mass-imbalanced homogeneous two-component Gross-Pitaevskii equation where each component has independent atom numbers and equal atomic masses. By changing the mass imbalance of our system we find that the shape of the vortices is deformed even at modest imbalances, leading to barrel-shaped vortices, which we quantify using a multicomponent variational approach. The energy of impurity carrying vortex pairs is computed, revealing a mass-dependent energy splitting. We then compute the excited states of the impurity, which we in turn use to construct "covalent bonds" for vortex pairs. Our work opens a route to simulating synthetic chemical reactions with superfluid systems.
\end{abstract}

DOI: 10.1103/PhysRevResearch.3.023085

\section{INTRODUCTION}

Quantized vortices represent the fundamental excitations of superfluids-atomic gases formed from interacting particles that exhibit nonviscous transport phenomena. Early experimental work revealed the nature of superfluid vortices [1-3] in these systems, which has also spurred theoretical interest, since the Gross-Pitaevskii formalism facilitates the accurate modeling of the phenomenology of superfluid alkali gases $[4,5]$.

Atomic Bose-Einstein condensates represent exceptionally pure physical systems, since it is possible to experimentally realize ground states with almost no noncondensate atoms present. This gives a unique opportunity to study the role of impurities in these systems, with recent pioneering experiments realizing both bosonic [6,7] and fermionic [8] polarons. Complementarily to this, experiments with binary condensates have now achieved the trapping of one matter wave inside another; in Ref. [9] a degenerate Fermi gas formed of ${ }^{6} \mathrm{Li}$ atoms was confined inside a Bose-Einstein condensate of ${ }^{133} \mathrm{Cs}$ atoms. Attractive atomic interactions provide another opportunity to understand the interaction of impurities with solitary waves in the nonlinear regime [10-12].

Homogeneous systems represent an important testing ground for theoretical ideas and have been under active experimental pursuit. Experiments with cold atomic gases typically use magnetic or optical traps to provide spatial confinement. However, recently it has become possible to confine atomic gases inside potentials that realize an effective box trap,

Published by the American Physical Society under the terms of the Creative Commons Attribution 4.0 International license. Further distribution of this work must maintain attribution to the author(s) and the published article's title, journal citation, and DOI. leading to homogeneous bosonic ground states in one[13,14], two- [15], and three-dimensional systems [16,17]. Degeneracy has also been achieved with Fermi gases confined in homogeneous potentials [18]. Homogeneous systems offer a unique opportunity to investigate superfluidity in a clean and precise way [19] and understand the nonequilibrium physics of uniform systems [20].

Quantum mechanical gases manifest superfluidity by the nucleation of quantized vortices when the gas undergoes rotation. Typically, this leads to the formation of the Abrikosov lattice at equilibrium; however, recent work has revealed that homogeneous [21], multi-component [22,23], and densitydependent [24] gauge theories all exhibit novel vortex configurations. Since individual vortices are topologically protected, they represent an intriguing candidate for hosting impurities within the matter wave [25]. In the context of the strongly interacting helium fluids, charged impurities in the form of electrons were originally proposed as an extension to the Gross-Pitaevskii formalism [26], which has recently found renewed interest due to the ability to accurately numerically simulate the Gross-Clark model [27].

The weakly correlated regime represented by atomic BoseEinstein condensates provides an important testing ground for understanding the dynamics of superfluid vortices [28], which in turn provides new theoretical $[29,30]$ and experimental [31] insight into quantum turbulence and nonequilibrium phenomenology [32]. Understanding the complex dynamics of superfluids is assisted by knowledge of the few-body dynamics of vortices; in particular, the realization of vortex dipoles [33] continues to provide important information [34,35], as well as the observation of solitonic vortices [36] in elongated superfluids. Multiple vortex states are generated by rotating the atomic cloud [37-39], leading at large rotation to the observation of unusual vortex dynamics [40,41].

Quantum mechanical gases with several coupled interacting internal degrees of freedom present an ongoing 
opportunity to gain deeper insight into exotic forms of superfluidity in microscopic systems [42]. Condensates with coherent couplings have also been an ongoing focus, with the dynamics of vortices in these models showing phenomenology analogous to high-energy physics [43-51]. Condensates with spin degrees of freedom have been shown to possess rich vortex physics, including stable multiply charged vortices $[52,53]$ in the ferromagnetic phase of these systems, attributed to the existence of both mass and spin current, leading to two individual forms of circulation. It has also been possible to consider "half"-quantized vortices in the antiferromagnetic phase, where the circulation is still quantized but the unit of quantization becomes a fraction leading again to unique lattice phases [54,55], as well as exotic vortex structures with nontrivial core structures, which have been studied in spinor condensates [56-61]. In multicomponent systems, vortices are usually coreless [62]: The cores of vortices in one component are filled by the other component(s). This leads to nontrivial vortex-vortex interactions $[63,64]$ and consequently rich phase structures of vortex lattices other than the Abrikosov vortex lattice $[22,47,62,65,66]$.

Multicomponent systems can also host more elaborate topological structures such as monopoles $[67,68]$ consisting of a radially varying spin structure, which have been realized in the ferromagnetic spin-1 phase of a ${ }^{87} \mathrm{Rb}$ condensate [69]. It is also possible to consider even more elaborate and exotic excitations, such as skyrmions in two dimensions (2D) [70] and 3D [71-75], which arise as a spatially varying spin deformation of the ground state of spinor systems as well as knots that are characterized in terms of the Hopf invariant, a topological charge unique to these excitations [76-78]. Multicomponent systems also host exotic composite topological excitations such as D-brane solitons-vortices ending on a domain wall [79-81]. Thus multicomponent superfluids provide an ongoing resource for topology, facilitating the creation of these exotic excitations in a stable and controlled environment.

We consider a two-component system in which only the first component is condensed, while the second feels the superfluid component as an effective potential. We study how impurity atoms can be trapped and manipulated by individual vortices and pairs of vortices in homogeneous superfluids. We specifically consider the situation where each component can have different atom numbers but the atomic masses of both components are equal. It is also possible to generalize this model to consider noninteracting fermionic impurities, which entails having a set of wavelike equations coupled to a superfluid component. Such a situation was proposed previously in Ref. [82], which modeled the experiment of Palzer et al. [83], who studied the transport properties of spin carrying impurity atoms through a one-dimensional Bose gas. By changing the number of atoms in the individual components, we reveal the unusual phenomenology of this system, including distorted vortex profiles and mass-dependent splitting of the impurities' energy. We then explore the properties of the excited states of the impurity, including the possibility of synthetic chemical bonds.

The paper is organized as follows. In Sec. II we introduce the model that describes our binary system, before examining the physics of individual vortices coupled to differing numbers of impurities in Sec. III, including a variational description of the system to explain the effect of the impurities on the vortex. We then compute the energy of pairs of vortices carrying impurities with different combinations of charges. Section IV examines the excited states of the vortex-impurity system, revealing the possibility of vortex chemistry with superfluids. In Sec. V we summarize our findings.

\section{THEORETICAL MODEL}

We consider a system of $N_{1}$ atoms forming a Bose-Einstein condensate described by the state $\psi_{1}$, self-consistently collisionally coupled to a second component of $N_{2}$ impurity atoms, described by the state $\psi_{2}$. We consider the case where both components have equal masses $M$. Then, after projecting the three-dimensional binary system into two dimensions by integrating out the axial degree of freedom, one obtains the coupled Gross-Pitaevskii equations

$$
\begin{aligned}
i \hbar \frac{\partial \psi_{1}}{\partial t} & =\left[-\frac{\hbar^{2}}{2 M} \nabla^{2}+g_{11}\left|\psi_{1}\right|^{2}+g_{12}\left|\psi_{2}\right|^{2}\right] \psi_{1}, \\
i \hbar \frac{\partial \psi_{2}}{\partial t} & =\left[-\frac{\hbar^{2}}{2 M} \nabla^{2}+g_{12}\left|\psi_{1}\right|^{2}\right] \psi_{2},
\end{aligned}
$$

where $g_{11}=\hbar^{2} a_{s} / M a_{\rho}^{2}$ encapsulates the scaled threedimensional $s$-wave scattering length $a_{s}$ of the atoms with mass $M$, while $g_{12}$ defines the strength of the collisional coupling between the superfluid and the impurity. The normalization of each component obeys $\int d \mathbf{r}\left|\psi_{j}(\mathbf{r})\right|^{2}=N_{j}$, while the mass imbalance is $N_{2} / N_{1}$. Meanwhile, the total (conserved) energy associated with the Gross-Pitaevskii model represented by Eqs. (1a) and (1b) is defined as

$$
E=\int d \mathbf{r}\left[\frac{\hbar^{2}}{2 M} \sum_{j}\left|\nabla \psi_{j}\right|^{2}+\frac{g_{11}}{2}\left|\psi_{11}\right|^{4}+g_{12}\left|\psi_{1}\right|^{2}\left|\psi_{2}\right|^{2}\right] .
$$

A single isolated two-dimensional vortex constitutes a hole on an otherwise homogeneous background, so it is important to separate the energy associated with the background and that of the vortex itself. As such we can write the total density of the superfluid as [84] $\left|\psi_{1}\right|^{2}=n_{1}^{(0)}-\left(n_{1}^{(0)}-\left|\psi_{1}\right|^{2}\right)$, where the background is $n_{1}^{(0)}$ (i.e., the value of the superfluid density far from the vortex core) while the term in parentheses accounts for the vortex core region. Then, the atom number for the superfluid component can be expressed as $N_{1}=\mathcal{A}_{2 \mathrm{D}} n_{1}^{(0)}-\int d \mathbf{r}\left(n_{1}^{(0)}-\left|\psi_{1}\right|^{2}\right)$, where $\mathcal{A}_{2 \mathrm{D}}$ defines the area of the first component. The atom number $N_{1}$ is composed of a constant background atom number $\mathcal{A}_{2 \mathrm{D}} n_{1}^{(0)}$ and the atom number of the vortex core. To obtain the energy of a single vortex in the two-component system, the energy associated with the homogeneous (vortex free) state is $E_{0} / n_{1}^{(0)}=g_{11} \mathcal{A}_{2 \mathrm{D}} n_{1}^{(0)} / 2-\int d \mathbf{r}\left[2\left(n_{1}^{(0)}-\left|\psi_{1}\right|^{2}\right)-g_{12}\left|\psi_{2}\right|^{2}\right]$, and by writing $E_{0} / n_{1}^{(0)}$, higher-order terms have been dropped, assuming that the size of the system $L_{x, y}$ satisfies $L_{x, y} \gg \xi$, where $\xi=\hbar / \sqrt{M \mu_{1}}$ is the size (healing length) of the vortex core. Then, the energy associated with the vortex $E_{\mathrm{v}+\mathrm{i}}=$ $E-E_{0}$ can be found using our expression for $E_{0}$ and Eq. (2), 
giving $E_{\mathrm{v}+\mathrm{i}}=\sum_{j=1}^{2} E_{j}^{\mathrm{kin}}+E_{11}^{\mathrm{vdW}}+E_{12}^{\mathrm{vdW}}$, where the individual contributions can be written as

$$
\begin{aligned}
E_{j}^{\mathrm{kin}} & =\frac{\hbar^{2}}{2 M} \int d \mathbf{r}\left|\nabla \psi_{j}\right|^{2}, \\
E_{11}^{\mathrm{vdW}} & =\frac{g_{11}}{2} \int d \mathbf{r}\left(\left|\psi_{1}\right|^{2}-n_{1}^{(0)}\right)^{2}, \\
E_{12}^{\mathrm{vdW}} & =g_{12} \int d \mathbf{r}\left(\left|\psi_{1}\right|^{2}-n_{1}^{(0)}\right)\left|\psi_{2}\right|^{2} .
\end{aligned}
$$

Equations (3a)-(3c) will be used in Sec. III to calculate the energy of different vortex configurations.

\section{NUMERICAL RESULTS}

To understand the physical behavior of the coupled superfluid-impurity system, we perform numerical simulations of the Gross-Pitaevskii model represented by Eqs. (1a) and (1b), subject to the constraint that the phase distribution $\vartheta_{1}(x, y)$ of the first component satisfies

$$
\vartheta_{1}(x, y)=\sum_{j=1}^{N_{v}} q_{j} \tan ^{-1}\left(\frac{y-y_{j}}{x-x_{j}}\right),
$$

which constitutes $N_{v}$ vortices of charge $q_{j}$ placed individually at $\left(x_{j}, y_{j}\right)$. In this paper we will take $q_{j}= \pm 1$. Although it is also possible to consider vortices of higher winding number, it is well known that such configurations are energetically unstable for the Gross-Pitaevskii model represented by Eqs. (1a) and (1b). We work in the healing units, where the healing length is defined as $\xi=\hbar / \sqrt{M n_{1} g_{11}}$, the units of energy are given in terms of $[E]=g_{11} n_{1}$, and time follows as $[T]=$ $\hbar / g_{11} n_{1}$. The wave functions are scaled as $\psi_{1} \rightarrow \sqrt{n_{1}^{(0)}} \psi_{1}$ and $\psi_{2} \rightarrow \psi_{2} / \xi$.

\section{A. Single vortices}

Figure 1 presents solutions of the Gross-Pitaevskii model represented by Eqs. (1a) and (1b) subject to the phase constraint of Eq. (4) for a single vortex with $q_{1}=+1$ [viz., Eq. (4)]. Throughout we have taken $N_{1}=4 \times 10^{3}$. Figures 1(a) and 1(b) present cross sections of the density of the vortex carrying superfluid $\left|\psi_{1}\right|^{2}$ and the impurity $\left|\psi_{2}\right|^{2}$ as a function of the number of impurity atoms $N_{2}$, respectively. The effect of changing the number of impurity atoms is quite dramatic: The shape of the vortex core is observed to change from the characteristic funnel shape presented in Fig. 1(i) showing the density and phase for $N_{2}=1$ to a barrel-shaped hole in the fluid, as presented in Fig. 1(ii) for $N_{2}=391$. Then, individual density cross sections are presented in Fig. 1(c) for the superfluid and impurity components. One can see the qualitative difference between the vortices' core shape (red dash-dotted curve) for $N_{1}=4 \times 10^{3}$ and $N_{2}=391$ (blue dashed curve). The corresponding impurity wave functions are plotted in the corresponding colors and styles; while the individual impurity densities are displayed in Figs. 1(d)-1(f) for $N_{2}=1,41,391$. Figure $1(\mathrm{~g})$ shows a calculation of the width (standard deviation) of the vortex core (black solid curve) and the impurity wave function (gray dashed curve).

\section{B. Variational calculation}

To build intuition and explore the physics of the vortex impurity model, we introduce a variational approach to understand the static properties of the mass-imbalanced system. As such we introduce three variational parameters, $\alpha, \beta$, and
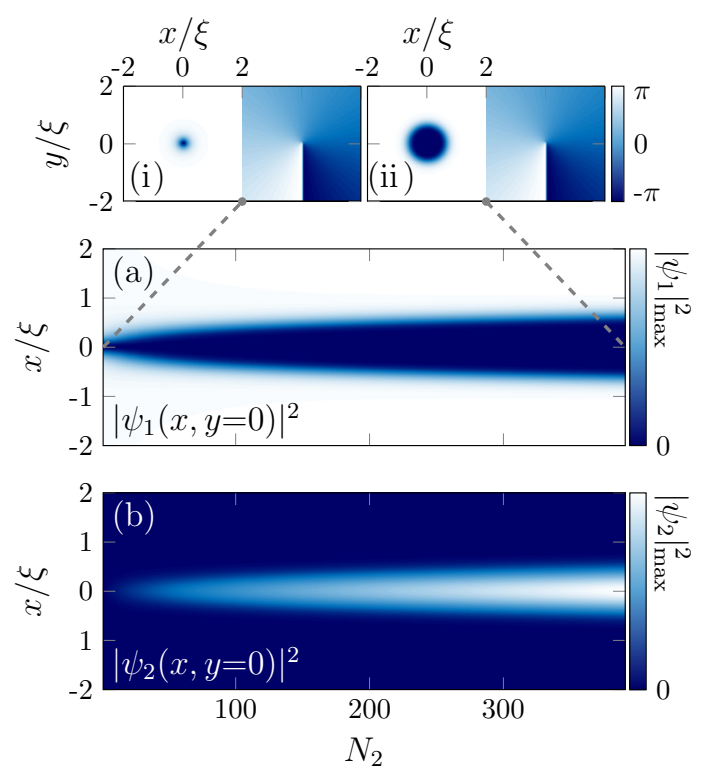
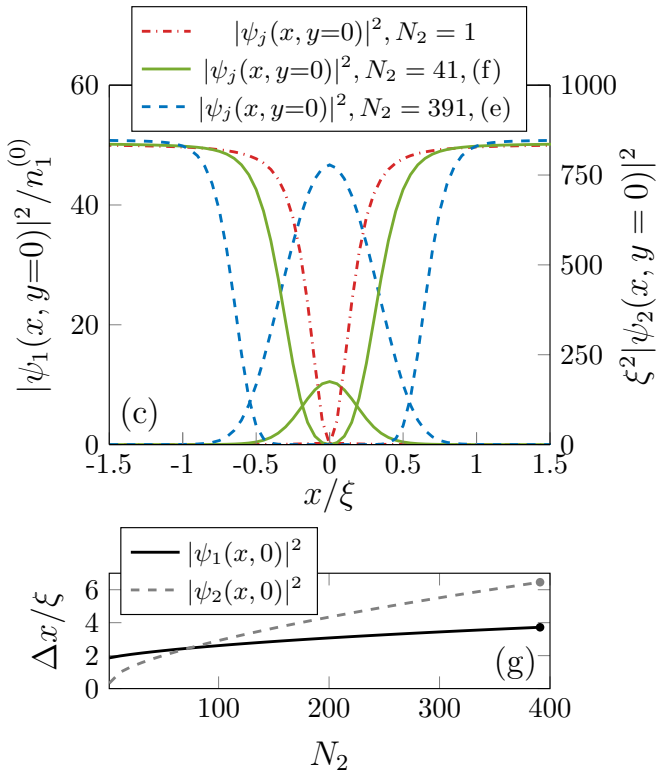

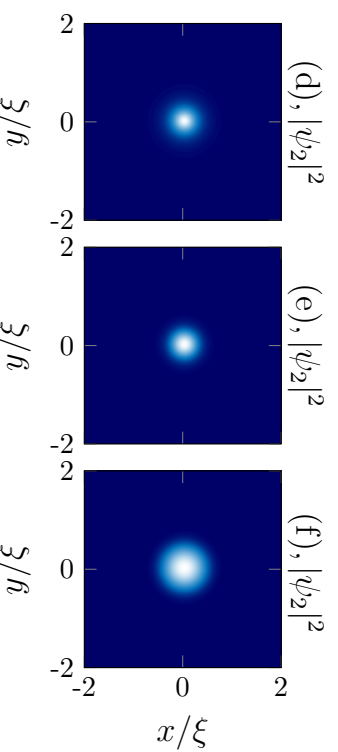

FIG. 1. Single vortex ground states. (a) and (b) show ground-state cross sections $\left|\psi_{j}(x, y=0)\right|^{2}$ of the superfluid and impurity as a function of $N_{2}$, respectively. Corresponding density $\left|\psi_{1}\right|^{2}$ and phase $\vartheta_{1}=\tan ^{-1}\left[\operatorname{Im}\left(\psi_{1}\right) / \operatorname{Re}\left(\psi_{1}\right)\right]$ distributions are displayed for $N_{2}=1$ and 391 in (i) and (ii). (c) shows the density cross section for each component for different numbers of impurities, while (d)-(f) show impurity densities $\left|\psi_{2}\right|^{2}$ for $N_{2}=\{1,41,391\}$, respectively. (g) shows the size (standard deviation) information of the superfluid-impurity system. Throughout we have taken $N_{1}=4 \times 10^{3}$. 
$\sigma$, which can be physically interpreted as the vortex length scale, inverse vortex radius, and impurity length scale, respectively. The choice of variational ansatz for the superfluid component can be thought of as a hybridization between logistic and Gaussian functions, which as we will demonstrate allows one to smoothly interpolate between a standard superfluid vortex-core profile and the unusual barrel-shaped configuration obtained for large numbers of impurity atoms presented in Fig. 1. Then a general-purpose variational ansatz (centered at the origin) describing the coupling between the two components $\psi_{1,2}^{\mathrm{var}}$ for a vortex of charge $q$ with varying mass imbalance $N_{2} / N_{1}$ will be taken as

$$
\begin{aligned}
& \psi_{1}^{\mathrm{var}}(x, y)=\sqrt{\frac{N_{c} \beta^{2}(\beta+1)}{\pi \alpha^{2}\left[(\beta-1) \ln \left(\frac{\beta}{\beta+1}\right)+1\right]}}\left[\frac{1}{\beta+\exp \left[-\left(x^{2}+y^{2}\right) / \alpha^{2}\right]}-\frac{1}{\beta+1}\right] \exp \left[i q \tan ^{-1}\left(\frac{y}{x}\right)\right], \\
& \psi_{2}^{\mathrm{var}}(x, y)=\sqrt{\frac{N_{2}}{\pi \sigma^{2}} e^{-\left(x^{2}+y^{2}\right) / 2 \sigma^{2}} .}
\end{aligned}
$$

The ansatz given by Eqs. (5a) and (5b) will be used in what follows to compute the variational energy of the system, as well as to obtain an approximate analytical expression for the angular momentum of the vortex. In order to compare the values of the energy obtained numerically from the GrossPitaevskii model represented by Eqs. (1a) and (1b) and the renormalized energy of Eqs. (3a)-(3c), we use the superfluid norm $N_{c}=\int d \mathbf{r}\left(n_{1}^{(0)}-\left|\psi_{1}^{\text {var }}\right|^{2}\right)$, which consequentially allows us to perform a semianalytic computation of the energy. Proceeding, we can substitute Eqs. (5a) and (5b) into Eqs. (3a)-(3c) to yield the individual contributions to the total variational energy $E_{\mathrm{v}+\mathrm{i}}^{\mathrm{var}}$, yielding the semianalytic expressions

$$
\begin{aligned}
E_{1, \mathrm{kin}}^{\mathrm{var}} & =N_{c} \frac{\hbar^{2}}{3 M \alpha^{2}} \frac{\beta+1}{(\beta-1) \ln \left(\frac{\beta}{\beta+1}\right)+1}\left\{\ln \left(\frac{\beta+1}{\beta}\right)-\frac{\beta}{(\beta+1)^{2}}+3 \beta^{2} q^{2} \int_{\xi_{v} / \alpha}^{R / \alpha} \frac{d u}{u}\left[\frac{1}{\beta+\exp \left(-u^{2}\right)}-\frac{1}{\beta+1}\right]^{2}\right\}, \\
E_{2, \mathrm{vin}}^{\mathrm{var}} & =N_{2} \frac{\hbar^{2}}{2 M \sigma^{2}}, \\
E_{11, \mathrm{vdW}}^{\mathrm{var}} & =N_{c}^{2} \frac{g_{11}}{12 \pi \alpha^{2}} \frac{6(\beta+1)(\beta-1)^{2} \ln \left(\frac{\beta+1}{\beta}\right)+1+3 \beta(3-2 \beta)}{(\beta+1)\left[(\beta-1) \ln \left(\frac{\beta}{\beta+1}\right)+1\right]^{2}}, \\
E_{12, \mathrm{vdW}}^{\mathrm{var}} & =\frac{2 g_{12} N_{c} N_{2}}{\pi \alpha^{2}} \frac{\beta^{2}(\beta+1)}{(\beta-1) \ln \left(\frac{\beta}{\beta+1}\right)+1} \int_{0}^{\infty} d u u\left[\left(\frac{1}{\beta+\exp \left(-\sigma^{2} u^{2} / \alpha^{2}\right)}-\frac{1}{\beta+1}\right)^{2}-\frac{1}{\beta^{2}(\beta+1)^{2}}\right] e^{-u^{2}} .
\end{aligned}
$$

To evaluate the various integrals that contribute to the total energy [Eqs. (3a)-(3c)], we can exploit the cylindrical symmetry of the problem and evaluate the energy in polar coordinates. The integrals required to obtain these results are listed in the Appendix. Note that the limits of integration (arising from the vortex phase) in Eq. (6a) require a little care. The lower limit is the (scaled) length scale of the vortex, the healing length $\xi_{v}$. This quantity can be approximated from the chemical potential of the homogeneous system, $\mu_{1}^{\mathrm{var}}=g_{11} n_{1}^{(0)}+g_{12} n_{2}^{(0)}$, where $n_{j}^{(0)}$ is the homogeneous density of each component, which can be computed using the variational wave function equations (5a) and (5b). Then $\xi_{v}=$ $\hbar / \sqrt{2 M \mu_{1}^{\mathrm{var}}}$. The upper limit $(\propto R)$ of this integral is simply the "radius" of this system, which can be obtained directly for a given simulation using $R=\sqrt{A_{\perp} / \pi}$, where $A_{\perp}$ is the area of the two-dimensional numerical box. We wish to compare the variational solutions for the energy, Eqs. (6a)-(6d), with the exact values computed using Eqs. (3a)-(3c) and the numerical solutions to the Gross-Pitaevskii model. We use the least-squares method to procure the variational parameters $\alpha$, $\beta$, and $\sigma$ for a given number of atoms $\left(N_{1}, N_{2}\right)$ and interaction strengths $g_{11}, g_{12}$. As such we wish to calculate

$$
\mathcal{F}_{j}=\min _{\alpha, \beta, \sigma} \sum_{k, l}\left\|\left.|| \psi_{j, \alpha, \beta, \sigma}^{\mathrm{var}}\left(x_{k}, y_{l}\right)\right|^{2}-\left|\psi_{j}^{\mathrm{num}}\left(x_{k}, y_{l}\right)\right|^{2}\right\|,
$$

where $\psi_{j}^{\text {num }}(x, y)$ are the numerical solutions obtained from the Gross-Pitaevskii model represented by Eqs. (1a) and (1b). Equation (7) can then be used to obtain a set of variational parameters for particular atom numbers and scattering lengths.

Figure 2 presents the solutions for $\alpha / \xi, 1 / \beta$, and $\sigma / \xi$ obtained from Eq. (7). The first two of these are the vortex variational parameters, plotted as $\alpha / \xi$ (blue crosses) and $1 / \beta$ (green triangles). One can see that the vortex "radius" $1 / \beta$ is less for small concentrations of impurity atoms and increases approximately linearly for larger numbers of impurities. The vortex length scale $\alpha / \xi$, on the other hand, increases for small concentrations of impurities, before leveling off at larger impurity numbers. There appears to be some correlation between the vortex length scale $\alpha / \xi$ and radius $1 / \beta$, which seems to occur for $N_{2} \simeq 100$, i.e., at the point where the length scale $\alpha / \xi$ levels off and the radius $1 / \beta$ starts to grow (gray dashed line). A possible explanation for this is that at this 


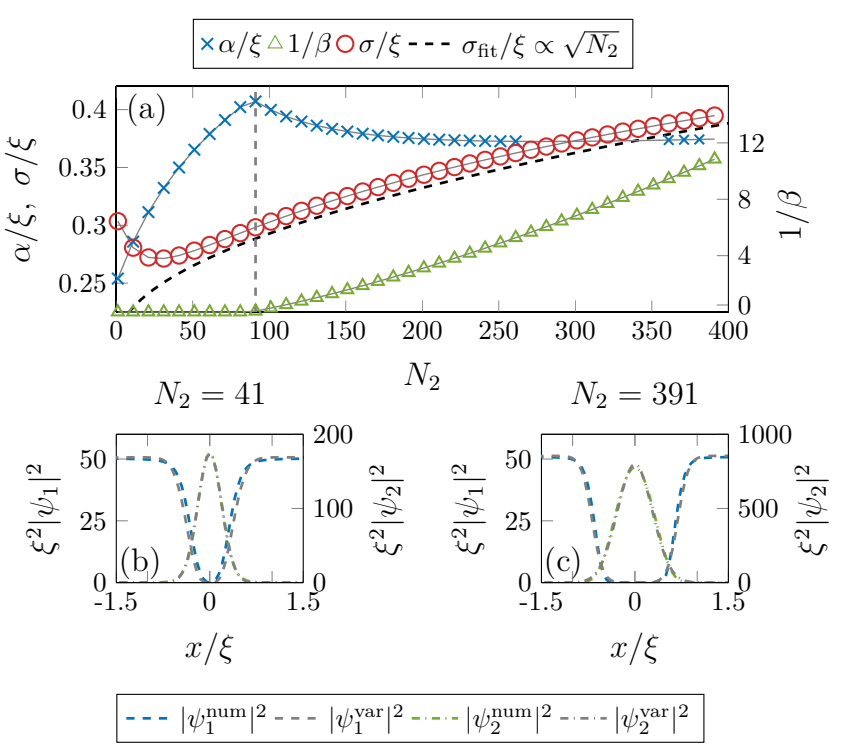

FIG. 2. Variational parameters. The variational parameters $\alpha / \xi$, $1 / \beta$, and $\sigma / \xi$ are shown as a function of the number of impurity atoms $N_{2}$, calculated from Eq. (7), in (a). The gray dashed line marks where the impurity starts to change the shape of the vortex (see text). Comparisons of the variational wave functions of Eqs. (5a) and (5b) with the numerical solutions of the Gross-Pitaevskii equations are shown in (b) and (c) for $N_{2}=41$ and 391, respectively.

concentration of impurity atoms the shape of the vortex starts to significantly change, developing the flat-bottomed profile [Fig. 1(c)], leading in turn to competition between the two terms appearing in the first fraction in square brackets in Eq. (5a). The impurity length scale $\sigma / \xi$ is also shown (open red circles). An accompanying fit is displayed (black dashed curve) to $\sigma_{\text {fit }} / \xi \propto \sqrt{N_{2}}$, which can be inferred from Eq. (6b), showing good agreement.

The expressions for the variational energy, Eqs. (6a)(6d), can be compared with the exact values found from the numerical solutions of the Gross-Pitaevskii model represented by Eqs. (1a) and (1b) and the renormalized energy of Eqs. (3a)-(3c). This is presented in Fig. 3. Figure 3(a) shows a comparison of the total variational energy (open green squares) and the total energy calculated from the exact numerical solutions (red pluses) for $\psi_{1,2}$ as a function of the number of impurity atoms, showing good agreement. Then, Figs. 3(b)-3(e) show the individual contributions to the total energy, while the two kinetic terms for the vortex and impurity, $E_{j, \text { kin }}$, are shown in Figs. 3(b) and 3(c), respectively, and the two interaction energies $E_{11, \mathrm{vdw}}$ and $E_{12, \mathrm{vdw}}$ are shown in Figs. 3(d) and 3(e), respectively. In general there seems to be good agreement between the two energies amongst the various contributions to the total energy, with the exception of the kinetic energy contribution, Fig. 3(b). This small difference likely originates from approximating the length scale $\xi_{v}$ of the vortex using the chemical potential of the homogeneous system.

The variational approach can also be used to calculate an expression for the angular momentum of the superfluid system. The expectation value of the $z$ projection of the angular momentum is defined as $\left\langle\hat{L}_{z}\right\rangle=\int d \mathbf{r} \psi_{\text {var }}^{*} \hat{L}_{z} \psi_{\text {var }}$. Using the representation $\psi_{\text {var }}=\sqrt{n_{\text {var }}} \exp \left(i \vartheta_{\text {var }}\right)$ to separate the density $n_{\text {var }}$ and phase $\vartheta_{\text {var }}$ variables, the angular momentum can then be computed asymptotically using Eq. (5a) and the assumption $R / \alpha \gtrsim 1$ as

$$
\frac{\left\langle\hat{L}_{z}\right\rangle_{\mathrm{var}}}{\hbar}=N_{c} \frac{R^{2} / \alpha^{2}-(\beta+1)+\left(\beta^{2}-1\right) \ln \left(\frac{\beta+1}{\beta}\right)}{(\beta+1)\left[(\beta-1) \ln \left(\frac{\beta}{\beta+1}\right)+1\right]} .
$$

The angular momentum of $\psi_{1}$ is calculated using three different approaches, which are compared in Fig. 3(f). The first two data sets show $\left\langle\hat{L}_{z}\right\rangle$ computed using the Gross-Pitaevskii solutions (blue pluses) and the variational angular momentum $\left\langle\hat{L}_{z}\right\rangle_{\mathrm{var}}$, Eq. (8) (open green circles). We can see that all three approaches are almost independent of $N_{2}$, as we would expect, and are only a few percent away from the theoretical value $\left\langle\hat{L}_{z}\right\rangle_{\text {Ana }}=\hbar N_{1}$ (red crosses).

\section{Vortex pairs}

As well as individual vortices, it is also useful to consider pairs of vortices: either with the same sign $\left(q_{1,2}= \pm 1\right)$ or with differing signs $\left(q_{1}=1, q_{2}=-1\right)$ [see Eq. (4)]. Then the

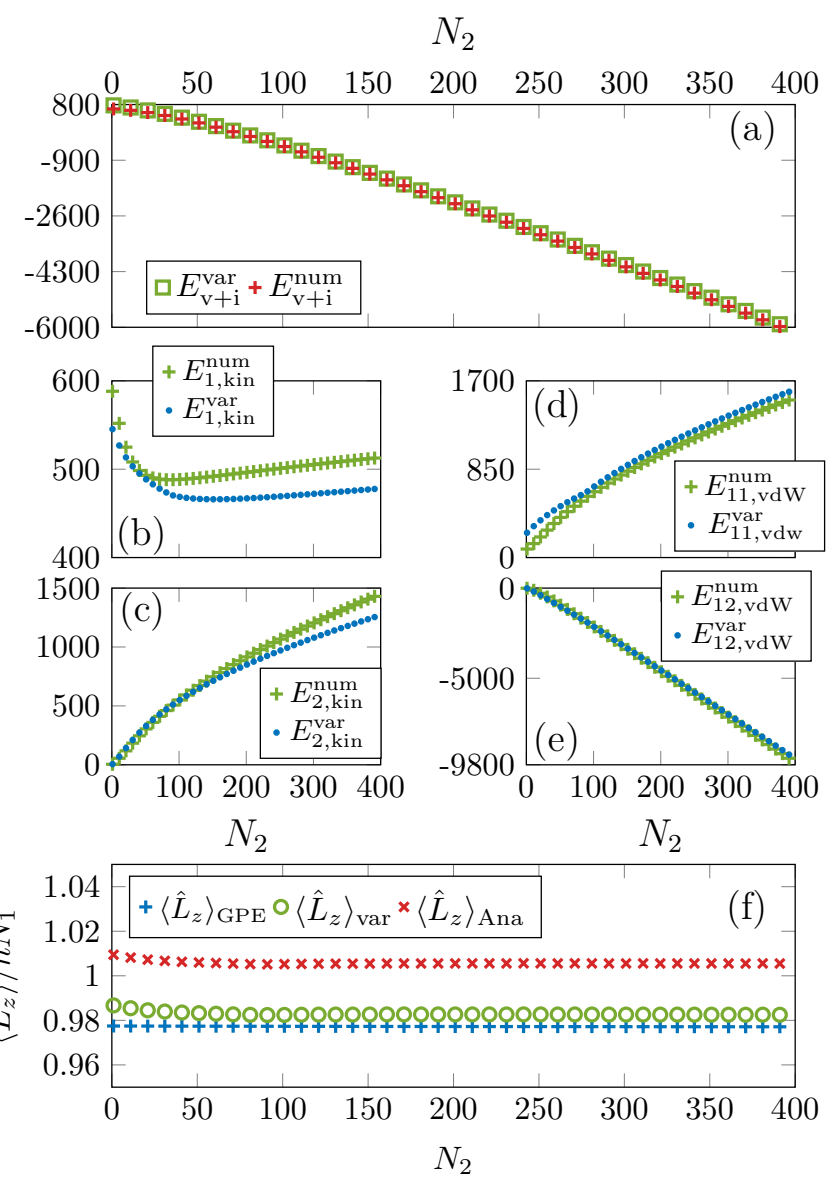

FIG. 3. Variational energy and angular momentum. The total variational energy and the total numerical energy are shown as a function of the number of impurity atoms $N_{2}$ in (a), while the different contributions to the variational energy, Eqs. (6a)-(6d), are shown in (b)-(e). The angular momentum $\left\langle\hat{L}_{z}\right\rangle$ is compared in (f), computed in three different ways. 
resulting phase distribution for the vortex pair is

$$
\vartheta_{1}(x, y)=q_{1} \tan ^{-1}\left[\frac{y}{x+d / 2}\right]+q_{2} \tan ^{-1}\left[\frac{y}{x-d / 2}\right],
$$

where the vortices are separated by a distance $x=d$. By changing the separation $d$ and mass imbalance ratio $N_{2} / N_{1}$, we can explore the effect that impurity atoms have. Our analysis is based on a static vortex configuration; hence it is instructive to consider when such an analysis is valid in the dynamical regime. A pair of like or opposite-sign vortices will corotate or propagate linearly, respectively. The angular frequency or velocity at which they do this depends in both cases on their relative separation. For two vortices placed in close proximity $(d \sim \xi)$, their motion will be faster relative to a larger separation $(d \gg \xi)$. In this paper it is this second regime that we will consider-hence additional contributions to the impurities' kinetic energy from the dynamics will be mitigated in this limit, and our analysis based on Eq. (9) is appropriate.

Figure 4 shows the computed impurity energies $E_{2}=$ $\int d \mathbf{r} \psi_{2}^{*}\left[-\left(\hbar^{2} / 2 M\right) \nabla^{2}+g_{12}\left|\psi_{1}\right|^{2}\right] \psi_{2} / N_{2}$ for different vortex charges and mass imbalances as a function of vortex separation. Note that the asymptotic energy $E_{2}(d \gg \xi)$ (i.e., the energy of two well-separated vortices) has been subtracted for a given atom number $N_{1}$ to aid comparison. At large mass imbalances $N_{2} / N_{1}=1 / 4 \times 10^{3}$ there is a small splitting between the like-sign and vortex dipole energies [blue data in Fig. 4(a)], while for larger imbalances the splitting is more pronounced \{e.g., $N_{2} / N_{1}=1 / 500$ [green data in Fig. 4(a)] and $N_{2} / N_{1}=1 / 250$ (red data) $\}$. We attribute this difference to the short-range nature of the vortices' densities, which for a vortex dipole manifest as a depression when the vortices approach, resulting in a lower impurity energy than for a pair of like-sign vortices. Then, Figs. 4(i)-4(iii) show example stationary states obtained from the solution of the GrossPitaevskii model represented by Eqs. (1a) and (1b). Here, Figs. 4(i) and 4(ii) show the density $\left|\psi_{1}\right|^{2}$ and phase $\vartheta_{1}$ of the first component, respectively, while Fig. 4(iii) shows the delocalized impurity density $\left|\psi_{2}\right|^{2}$. These calculations reveal a simple relationship between the mass imbalance $N_{2} / N_{1}$ and the splitting of the impurities' energy, with smaller mass imbalances leading to larger differences in the impurities' energy at short separations. Since we know that a vortex pair's energy is logarithmic in their separation $d$ such that $E_{12} \propto q_{1} q_{2} \ln (R / d)$, larger vortices will comparatively feel an increased repulsion or attraction at the same distance $d$ compared with those with smaller core sizes, consistent with the known behavior of vortices. Figure 4 demonstrates that the presence of impurities will contribute an attractive interaction between vortices.

To investigate the dynamical behavior of the energy $E_{2}$ for $T>0$, we can evolve the Gross-Pitaevskii model represented by Eqs. (1a) and (1b) in time to some later time $T$ and recalculate the energies using the new $\psi_{1,2}(x, y, T)$ of the superfluid and impurity components. In Figs. 5(a)-5(c) the energies $E_{1}(T), E_{2}(T)$, and $E(T)$, respectively, of vortex pairs are presented for $N_{1}=4 \times 10^{3}, N_{2}=1$ with vortex charges $q_{1}=q_{2}=+1$ for $T=0,0.5,1,2$. Accompanying this are six examples of the vortices' and impurities' densities at times

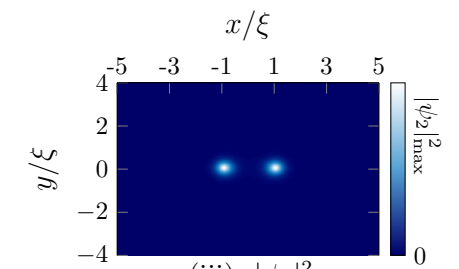

(iii), $\left|\psi_{2}\right|^{2}$
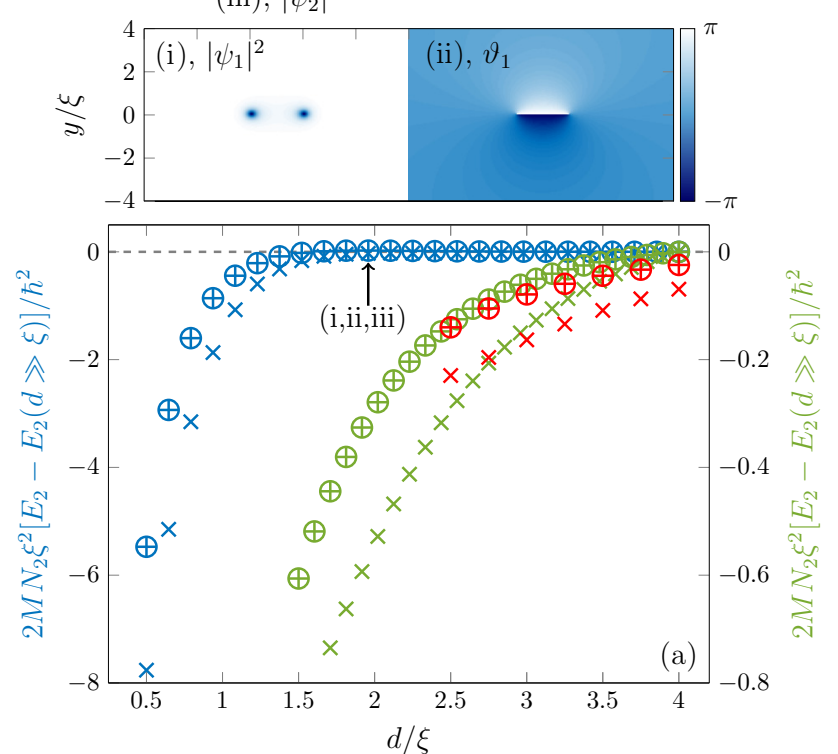

$$
\begin{aligned}
& +\quad q_{1,2}=1, N_{1}=4 \times 10^{3} \\
& \text { ○ } \quad q_{1,2}=-1, N_{1}=4 \times 10^{3} \\
& \times q_{1}=1, q_{2}=-1, N_{1}=4 \times 10^{3} \\
& \begin{array}{l}
+\quad q_{1,2}=1, N_{1}=5 \times 10^{2} \\
\bigcirc \quad q_{1,2}=-1, N_{1}=5 \times 10^{2} \\
\times \\
q_{1}=1, q_{2}=-1, N_{1}=5 \times 10^{2} \\
+\quad q_{1,2}=1, N_{1}=2.5 \times 10^{2} \\
\bigcirc \quad q_{1,2}=-1, N_{1}=2.5 \times 10^{2} \\
\times q_{1}=1, q_{2}=-1, N_{1}=2.5 \times 10^{2}
\end{array}
\end{aligned}
$$

FIG. 4. Vortex pair energies. (a) shows the spectral flow (eigenvalues) $E_{2}-E_{2}(d \gg \xi)$ of the impurity for different combinations of vortex charges [Eq. (9)] and mass imbalances for a single impurity $N_{2}=1$. Examples of the stationary states are shown in (i)-(iii) corresponding to a vortex dipole with $N_{1}=4 \times 10^{3}$ [see black arrow in (a)]. Note that the left-axis data in (a) correspond to the left legend, while the right-axis data correspond to the right legend.

$T=0,1,2$. The vortices' displacement is $d=2 \xi$. Then for $T>0$ the anticlockwise rotation direction is indicated by $\Omega_{\mathrm{Mag}}$. Figure 5(b) shows the important time-dependent impurity energies, $2 M N_{2} \xi\left[E_{2}-E_{2}(d>>\xi)\right] / \hbar^{2}$, for separations in the range $d / \xi=2: 4$. Already even modest separations show that this quantity does not change appreciably during dynamics, while at larger separations there is almost no difference from the static case. Complementarily to this, since we consider the limit $N_{2} / N_{1} \ll 1$, the effect of excitations such as sound waves from the impurity on the superfluid component will be minimal, since fluctuations will scale as $\sqrt{N_{1} N_{2}}$, which would be subleading compared with those originating from interactions between superfluid atoms in the first component. The calculations presented here support the use of static vortex configurations to investigate the properties of vortex-trapped impurities in this manner. 


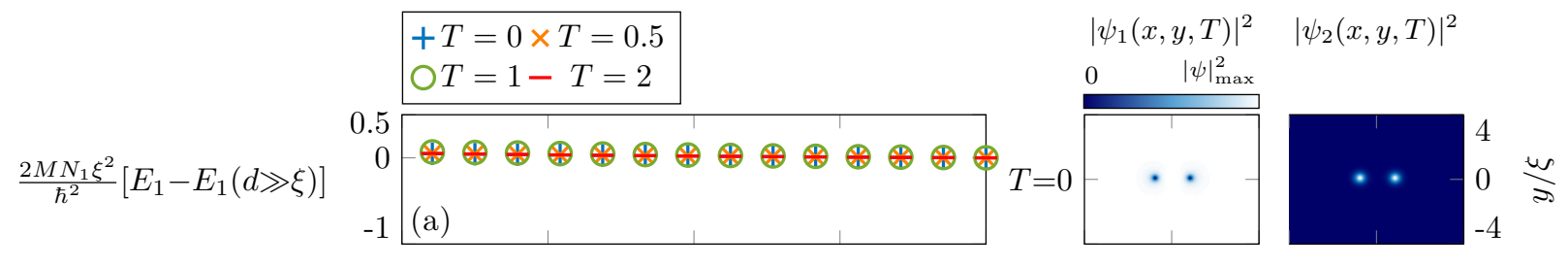

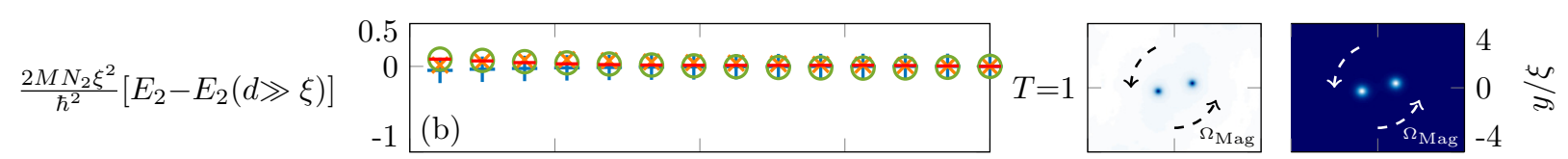

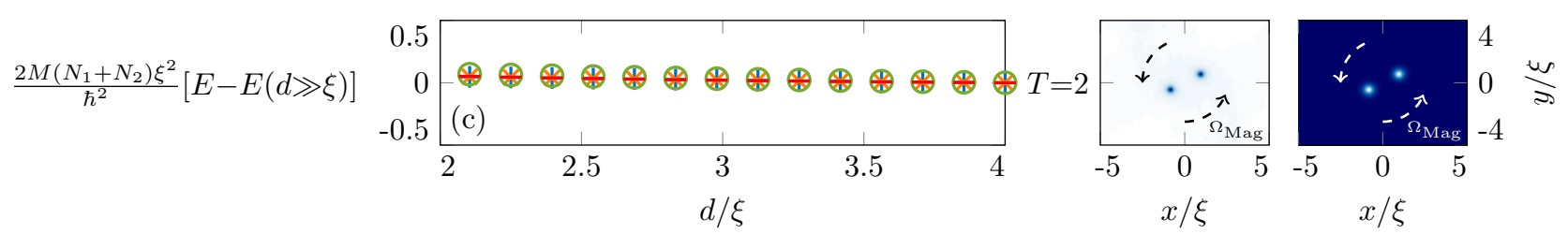

FIG. 5. Vortex-impurity dynamics. (a)-(c) show the energies $E_{1}$ and $E_{2}$ and the total energy $E$ as a function of displacement $d / \xi$ for $T=0,0.5,1,2$. The six accompanying density plots $|\psi(x, y, T)|^{2}$ show examples of dynamics for the case of corotation, $q_{1}=q_{2}=+1$ with $d / \xi=2$.

\section{COVALENT BONDS BETWEEN WELL-SEPARATED VORTICES}

In this section we consider the possibility of bonding between a pair of like-sign vortices. Motivated by our findings in the previous section, where the interaction between two well-separated vortices with both like and different signs were considered, we consider how the excited states of such a system can contribute an additional attractive force. Note that the covalent force we will study in this section is the next-to-leading order interaction [the leading interaction is $\sim \ln (d / \xi)$ ]. Also, the well-separated vortices corotate or comove with their separation kept constant, so that the intervortex forces do not change appreciably in time. Hence we will deal with only static vortices in this section.

\section{A. Padé approximation for a single vortex}

Let us begin by writing the time-independent form of the Gross-Pitaevskii model represented by Eqs. (1a) and (1b) using the transformation $\psi_{j}(\mathbf{r}, t)=\exp \left(-i N_{j} E_{j} t / \hbar\right) \phi_{j}(\mathbf{r})$, giving

$$
\begin{gathered}
{\left[-\frac{\hbar^{2}}{2 M} \nabla^{2}+g_{11}\left|\phi_{1}\right|^{2}+g_{12}\left|\phi_{2}\right|^{2}\right] \phi_{1}=N_{1} E_{1} \phi_{1},} \\
{\left[-\frac{\hbar^{2}}{2 M} \nabla^{2}+g_{12}\left|\phi_{1}\right|^{2}\right] \phi_{2}=N_{2} E_{2} \phi_{2} .}
\end{gathered}
$$

A dimensionless set of units can be adopted by scaling the units of length by the healing length $\xi, \phi_{1} \rightarrow \sqrt{n_{1}} \phi_{1}$ and $\phi_{2} \rightarrow \phi_{2} / \xi$. We then perform one additional scaling, $\phi_{j} \rightarrow$ $\sqrt{N_{j}} \phi_{j}$, which allows us to understand the parameter regimes in terms of the coupling between the superfluid and the impurity. This yields the coupling parameters [corresponding to the dimensionless coefficients of $\left|\phi_{1}\right|^{2}$ and $\left|\phi_{2}\right|^{2}$ in Eqs. (11) and (10), respectively] as $C_{1}=2 M g_{12} \xi^{2} n_{1} N_{j} / \hbar^{2}$ and $C_{2}=2 M g_{12} N_{2} / \hbar^{2}$, while the units of energy are scaled as $2 M N_{j} \xi^{2} E_{j} / \hbar^{2}$ for the energy of component $j$. The ratio of coupling constants yields $C_{2} / C_{1}=\left(N_{2} / N_{1}\right)\left(1 / n_{1} \xi^{2}\right)$, which in the limit $N_{1} \gg N_{2}$ allows us to decouple Eqs. (10) and (11). One can also consider the case of unequal masses $M_{1} \neq M_{2}$, in which case this condition becomes instead $\left(M_{1} N_{2} / M_{2} N_{1}\right)\left(1 / n_{1} \xi^{2}\right) \ll 1$, allowing additional parameter flexibility in studying the impurity physics of the model of Eqs. (10) and (11). Then, by separating the phase and amplitude of the vortex as $\phi_{1}=\sqrt{\frac{N_{1} E_{1}}{g_{11}}} e^{i q \theta} f(\rho)$ we obtain a simplified form of Eq. (10),

$$
-\xi^{2}\left(\frac{d^{2} f}{d \rho^{2}}+\frac{1}{\rho} \frac{d f}{d \rho}-\frac{f}{\rho^{2}}\right)+2\left(f^{2}-1\right) f=0 .
$$

To build the Padé approximation, we can solve Eq. (12) in two limits subject to the boundary conditions $f(\rho \rightarrow 0)=0$ and $d f(\rho) /\left.d \rho\right|_{\rho \rightarrow \infty}=0$. This gives the pair of asymptotic solutions

$$
\begin{gathered}
f(\rho \rightarrow 0)=a \rho / \xi+\mathcal{O}\left(\rho^{3}\right), \\
f(\rho \rightarrow \infty)=1-\frac{\xi^{2}}{4 \rho^{2}}+\mathcal{O}\left(\rho^{-4}\right) .
\end{gathered}
$$

The constant $a$ appearing in Eq. (13) is the first fitting constant used to build the Padé approximation [85]. We note that the authors of Ref. [25] used a piecewise function to perform a variational analysis of the vortex's allowed bound states. Such an approach is useful for analytical treatments; however, since we wish to obtain the excited states numerically, the Padé approximation provides an accurate approximation to the numerical solution of the Gross-Pitaevskii model and hence a closer agreement to the true numerical spectrum of the impurity. Then, an appropriate approximation for a single vortex is written as

$$
f^{P}(\rho)=\sqrt{\frac{a^{2}(\rho / \xi)^{2}+2\left(b^{2}-a^{2}\right)(\rho / \xi)^{4}}{1+b^{2}(\rho / \xi)^{2}+2\left(b^{2}-a^{2}\right)(\rho / \xi)^{4}}},
$$

which satisfies both asymptotic boundary conditions given by Eqs. (13) and (14). Figure 6 shows a comparison between the numerical solutions to Eq. (12) (blue solid curve) and the Padé 


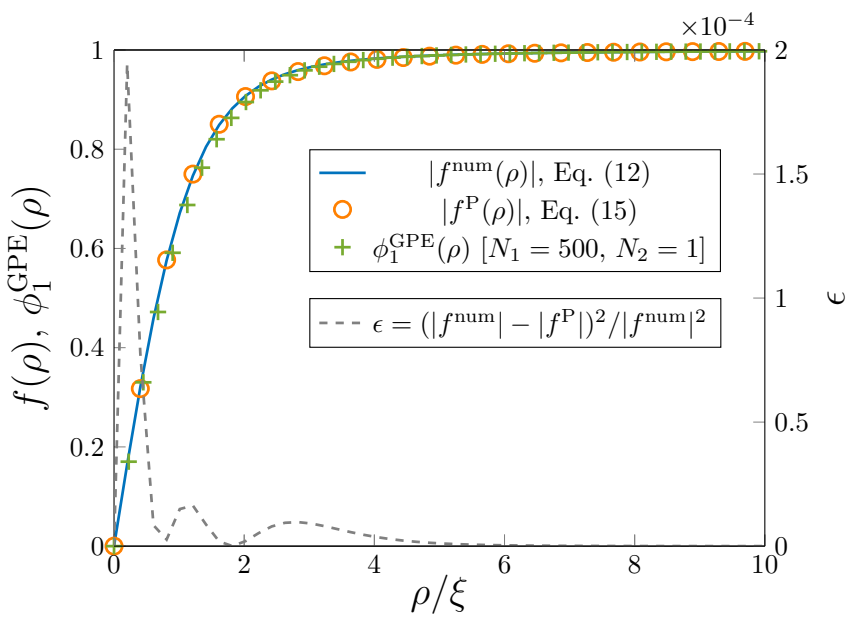

FIG. 6. Padé approximation. The vortex solution $\left|f^{\text {num }}(\rho)\right|$ to Eq. (12) (blue solid curve) and the accompanying Padé approximation, $\left|f^{P}(\rho)\right|$, of Eq. (15) (open orange circles) are shown (left vertical scale). The scaled solution $\phi_{1}^{\mathrm{GPE}}(\rho)$ to Eqs. (10) and (11) with $N_{1}=500, N_{2}=1$ is shown as green pluses. The scaled difference $\epsilon=\left(\left|f^{\text {num }}\right|-\left|f^{P}\right|\right)^{2} /\left|f^{\text {num }}\right|^{2}$ (gray dashed curve) is shown on the right vertical scale.

approximation, Eq. (15) (open orange circles). The two fitting parameters are found to be $a \sim 0.81$ and $b \sim 0.88$. Accompanying this, the (scaled) solution $\phi_{1}^{\mathrm{GPE}}$ obtained from Eqs. (10) and (11) with $N_{1}=500, N_{2}=1$ and $C_{2}=2 M g_{12} N_{2} / \hbar^{2}=$ 1 is shown for comparison (left vertical axis). The dashed gray curve shows the difference $\epsilon=\left(\left|f^{\text {num }}\right|-\left|f^{P}\right|\right)^{2} /\left|f^{\text {num }}\right|^{2}$ (right vertical axis) between the numerical and Padé approximations, which being small, supports this choice.

\section{B. Impurity potential model}

Having the analytic Padé approximation of Eq. (15) for $f^{P}(\rho)$, we can proceed to solve Eq. (11). Since we assume that $\left(N_{2} / N_{1}\right)\left(1 / \xi^{2} n_{1}\right)$ is small, we can treat $\phi_{1}$ in Eq. (11) as an external potential by omitting any back reaction between the vortex and the impurity. We are left with the two-dimensional quantum mechanical problem of calculating the bound states of $\phi_{2}$ of an impurity by the axially symmetric Schrödinger potential $g_{12} f^{P}(\rho)^{2}$. Then we have

$$
\left[-\frac{\hbar^{2}}{2 M} \nabla^{2}+\mathcal{U}(\rho)\right] \phi_{2}=\mathcal{E} \phi_{2} .
$$

Here, the potential $\mathcal{U}(\rho)=g_{12}\left(\left|\phi_{1}^{P}\right|^{2}-N_{1} E_{1} / g_{11}\right)$ satisfies $\mathcal{U}(\rho \rightarrow \infty)=0$, while the energy is $\mathcal{E}=N_{2} E_{2}-$ $N_{1} E_{1} g_{12} / g_{11}$. Then, the Schrödinger potential $\mathcal{U}(\rho)$ obeys $\mathcal{U}(0)=-N_{2} E_{2} g_{12} / g_{11}, \mathcal{U}(\rho \rightarrow \infty)=0$; hence the potential exists on the interval $-N_{2} E_{2} g_{12} / g_{11} \leqslant \mathcal{U}(\rho)<0$. Since the potential $\mathcal{U}(\rho)$ is axisymmetric, we can separate $\rho$ and $\theta$ as

$$
\phi_{2}(\rho, \theta)=\sqrt{\frac{N_{2} E_{2}}{g_{11}}} \frac{\varphi(\rho)}{\sqrt{\rho}} \exp (\operatorname{im} \theta) .
$$

Here, $m \in \mathbb{Z}$ defines the quantum number of angular momentum for the impurity, with the associated operator $\hat{L}_{z}=$ $-i \partial_{\theta}$. Normalization for $\varphi(\rho)$ is then imposed through $2 \pi \int_{0}^{\infty} d \rho \varphi(\rho)^{2}=N_{2}$. Inserting Eq. (17) into Eq. (16), we are left with the following one-dimensional Schrödinger problem:

$$
\left[\hat{Q}^{\dagger} \hat{Q}+m^{2} \frac{\hbar^{2}}{2 M \rho^{2}}+\mathcal{U}(\rho)\right] \varphi_{n, m}=\mathcal{E}_{n, m} \varphi_{n, m},
$$

where we have introduced the operator

$$
\hat{Q}=\frac{\hbar}{\sqrt{2 M}}\left(-\frac{d}{d \rho}+\frac{1}{2 \rho}\right) \text {. }
$$

Then we have $\hat{Q}^{\dagger} \hat{Q}=-\left(\hbar^{2} / 2 M\right)\left(d^{2} / d \rho^{2}+1 / 4 \rho^{2}\right)$, while the term $m^{2} \hbar^{2} / 2 M \rho^{2}$ in Eq. (18) defines the centrifugal potential felt by the impurity. To understand the basic properties of the impurity, let us first consider $m=0$. Note that the operator $\hat{Q}^{\dagger} \hat{Q}$ is positive semidefinite. Therefore, when $\mathcal{U}(\rho)$ vanishes $\left(g_{12} \rightarrow 0\right)$, the eigenvalue $\mathcal{E}$ satisfies $\left.\mathcal{E}\right|_{g_{12} \rightarrow 0} \geqslant 0$. The lowest eigenstate $(n=1)$ has $\mathcal{E}=0$ and should satisfy $\hat{Q} \varphi=0$. This can be easily solved by $\varphi(\rho) \propto \sqrt{\rho}$. This in turn corresponds to constant $\phi_{2}$, which is clearly nonnormalizable. Now we consider small but finite $g_{12}$ by slightly increasing $g_{12}$ from zero. This has the effect of lowering the Schrödinger potential $\mathcal{U}(\rho)$ everywhere since $\mathcal{U}(\rho)<0$. Then, because we can continuously change $g_{12}$ from 0 to $g_{12}>0$, there is a corresponding continuous shift of the eigenvalues. Namely, the ground-state energy should satisfy $\mathcal{E}_{1,0}<0$ for $g_{12} \neq 0$. Therefore the Schrödinger equation at $\rho \rightarrow \infty$ reads $d^{2} \varphi_{1,0} / d \rho^{2}=2 M\left|\mathcal{E}_{1,0}\right| \varphi_{1,0} / \hbar^{2}$, the solution for which is a bound state whose asymptotic wave function is

$$
\varphi_{1,0}(\rho) \propto \exp \left(-\sqrt{2 M\left|\mathcal{E}_{1,0}\right|} \rho / \hbar\right) .
$$

To summarize, we have shown that there exists at least one bound state regardless of the form of the potential $\mathcal{U}(\rho)$. On the other hand, the existence of (bound) excited states $\varphi_{n, 0}(\rho)$ for $n>0$ sensitively depends on $g_{12}$. In general the greater $g_{12}$ is, the more excited states exist and hence there are more possibilities to form bound states with a particular potential. On the other hand, for $m \neq 0$, the centrifugal potential always lifts the Schrödinger potential since it contributes a positive energy to the total potential felt by the impurity. Therefore a sufficiently large $g_{12}$ is required to accommodate bound states for $\varphi_{n, 0}(n>1)$.

\section{Single vortex bound states}

Let us next numerically obtain the eigenvalues. Having the analytic approximation $f^{P}(\rho)$ in hand is an advantage for this purpose. We choose two examples $C_{1}=2 M g_{12} \xi^{2} n_{1} N_{1} / \hbar^{2}=$ 1 and $C_{1}=5$ as demonstrations. For the small $C_{1}=1$, there exists only one ground state $(n, m)=(1,0)$ with the energy $2 M N_{2} \xi^{2} \mathcal{E}_{1,0} / \hbar^{2} \simeq-0.25$. We show a two-dimensional plot of $\phi_{2}^{1,0}$ in Fig. 7. For the large $C_{1}=5$ the potential is deeper, so that we have six bound states with $(n, m)=(1,0),(1,1),(2,0),(1,2),(2,1),(3,0)$. The corresponding energy eigenvalues $2 M N_{2} \xi^{2} \mathcal{E}_{n, m} / \hbar^{2}$ are -2.85 , $-1.21,-0.46,-0.18,-0.09$, and -0.03 , respectively. The corresponding number of circular nodes is given by $n-1$, and that of linear nodes is $|m|$. Then, the total number of nodes is given by $N=n+|m|-1$. Therefore $(1,1)$ and $(2,0)$ have a single node, and we observe $\mathcal{E}_{1,1}<\mathcal{E}_{2,0}$. Similarly, $(1,2)$, $(2,1)$, and $(3,0)$ have double nodes, and the corresponding energies obey $\mathcal{E}_{1,2}<\mathcal{E}_{2,1}<\mathcal{E}_{3,0}$. From these observations, we 


\begin{tabular}{|c|c|c|c|c|c|c|c|}
\hline & $C_{1}=1$ & \multicolumn{6}{|c|}{$C_{1}=5$} \\
\hline $\mathcal{E}$ & -0.253884 & -2.84518 & -1.20557 & -0.459918 & -0.18152 & -0.0904204 & -0.0301221 \\
\hline & & & $\bullet$ & & $\&$ & c.) & \\
\hline & $\bullet$ & - & & ○ & & & ○) \\
\hline & & & $:$ & & $\mathscr{Z}$ & & \\
\hline$(n, m)$ & $(1,0)$ & $(1,0)$ & $(1,1)$ & $(2,0)$ & $(1,2)$ & $(2,1)$ & $(3,0)$ \\
\hline orbitals & $1 \mathrm{~s}$ & $1 \mathrm{~s}$ & $2 p$ & $2 \mathrm{~s}$ & $3 \mathrm{~d}$ & $3 p$ & $3 \mathrm{~s}$ \\
\hline
\end{tabular}

FIG. 7. The impurity wave functions $\phi_{2}^{1 s}$ for $C_{1}=2 M g_{12} \xi^{2} n_{1} N_{1} / \hbar^{2}=1$, and $\phi_{2}^{1 s}, \phi_{2}^{2 p}, \phi_{2}^{2 s}, \phi_{2}^{3 d}, \phi_{2}^{3 p}$, and $\phi_{2}^{3 s}$ for $C_{1}=5$ are shown. The red color means $\phi_{2}^{m, n}>0$, while the blue corresponds to $\phi_{2}^{m, n}<0$ and the white corresponds to $\phi_{2}^{n, m}=0$. The plot region is $x / \xi \in[-10,10]$ and $y / \xi \in[-10,10]$.

see that creating a circular node requires more energy than a linear one. Figure 7 shows $\phi_{n, m}$ for the six bound states. We adopt the conventional names for atomic orbitals. For example, the bound state $(n, m)=(1,0)$ is called the $1 \mathrm{~s}$ orbital because the first number 1 corresponds to $N+1=1$ while $\mathrm{s}$ is for $|m|=0$. Then we can adopt the conventional rules, $(1,1)=2 p,(2,0)=2 s,(1,2)=3 d,(2,1)=3 p,(3,0)=$ $3 s$, etc. Note that by increasing $2 M g_{12} \xi^{2} n_{1} N_{1} / \hbar^{2}$ from 1 to 5 , the potential depth becomes deeper while its width is unchanged. Accordingly, the wave functions for $C_{1}=5$ are more compact than those of $C_{1}=1$. We note that the authors of Ref. [25] also studied the excited states of a single impurity confined by a vortex; however, our approach differs somewhat since we will in the remaining sections demonstrate how these states can be used to build an analogy with chemical bonds.

\section{Covalent bonds between two vortices}

In this section we compute the excited states of two neighboring vortices. We consider two vortices placed at $(x, y)=$ $( \pm d / 2,0)$. For the background configuration of $\phi_{1}$, we take a product ansatz

$$
\phi_{1}^{(2)}(x, y, d)=\sqrt{\frac{g_{11}}{N_{1} E_{1}}} f^{P}\left(x-\frac{d}{2}, y\right) f^{P}\left(x+\frac{d}{2}, y\right),
$$

and we solve Eq. (16) with the replacement $\mathcal{U}(\rho) \rightarrow$ $g_{12}\left(\left|\phi_{1}^{(2)}\right|^{2}-N_{1} E_{1} / g_{11}\right)$. The presence of one vortex affects the other vortex, and the distortion cannot be ignored when the separation $d$ is small. Therefore, in what follows, we will take $d \gg \xi$ for the above product ansatz to be a valid approximation. As explained in Sec. IV B, we omit any back reaction between the field $\phi_{1}$ and $\phi_{2}$ by keeping $\left(N_{2} / N_{1}\right)\left(1 / \xi^{2} n_{1}\right) \ll 1$. In Sec. IV C, it was explained that each background $\phi_{1}$ vortex represents an attractive potential for the impurity atoms. Therefore, when we place two such vortices on the plane, the impurities are attracted by such a pair of vortices. However, when the displacement $d \gg \xi$, the individual vortex wave functions no longer overlap, so the energy spectra are the same as those of a single vortex, $2 M N_{2} \xi^{2} \mathcal{E}_{1 s}^{(1)} / \hbar^{2} \simeq-0.25$. On the other hand, when the vortex separation $d$ is large but finite, the degeneracy is lifted, and instead the spectra are slightly split. Since the problem is no longer axially symmetric, we consider instead Eq. (11) with $\phi_{1}$ replaced by $\phi_{1}^{(2)}$, from which we obtain the numerical solutions. Let us start with the simple case of $C_{1}=1$, for which each vortex can have only a single $1 s$ orbital. We vary $d / \xi$ from $d=3 \xi$ to $d=12 \xi$ with step size $\delta d=\xi / 2$. For each given $d$, we numerically solve Eq. (11) and determine the energy eigenvalues. The results are summarized in Fig. 8. As expected we have two states: a state with even symmetry, while the other is an odd function for the parity $x / \xi \rightarrow-x / \xi$. The former is the ground state, whereas the latter is the excited state, since it has one linear node on the $y$ axis. The two states are almost degenerate when $d$ is sufficiently large (see $d \gtrsim 10 \xi$ of Fig. 8). These states lie slightly below the ground-state energy of the single vortex, $\mathcal{E}_{1 s}^{(1)}$, and for large but finite $d$, they asymptotically approach $\mathcal{E}_{1 s}^{(1)}$ from below. Therefore, at asymptotically large $d$, having impurities for both the ground state and the excited state results in an attractive force between the two background vortices of $\phi_{1}$. By decreasing $d(\lesssim 9 \xi)$, however, the ground and the first excited states show different behaviors. The groundstate energy is a monotonically decreasing function of $d$ in the limit $d \rightarrow 0$. The decrease in the ground-state energy occurs because the impurity wave function can localize around both of the vortices and its length scale increases compared with the single vortex background. Namely, sharing the impurity between two vortices gives rises to an attractive force. This is nothing but a covalent-bonding-like effect for the vortices. Since the $(1,0)$ wave function is axially symmetric, we may call this the $\sigma_{s-s}$ bond. Then, we find a numerical fit of the covalent energy of the ground state as a function of $d(\gtrsim 5 \xi)$ as

$$
\mathcal{E}_{(1,0)+(1,0)}(d) \simeq \mathcal{E}_{1,0}^{(1)}-11.57 \frac{\hbar^{2}}{2 M N_{2} \xi^{2}}\left(\frac{\xi}{d}\right)^{3.49} .
$$

On the other hand, the energy eigenvalues of the first excited state increase as $d$ is decreased. This is the so-called antibonding effect common for states of odd parity. The presence 


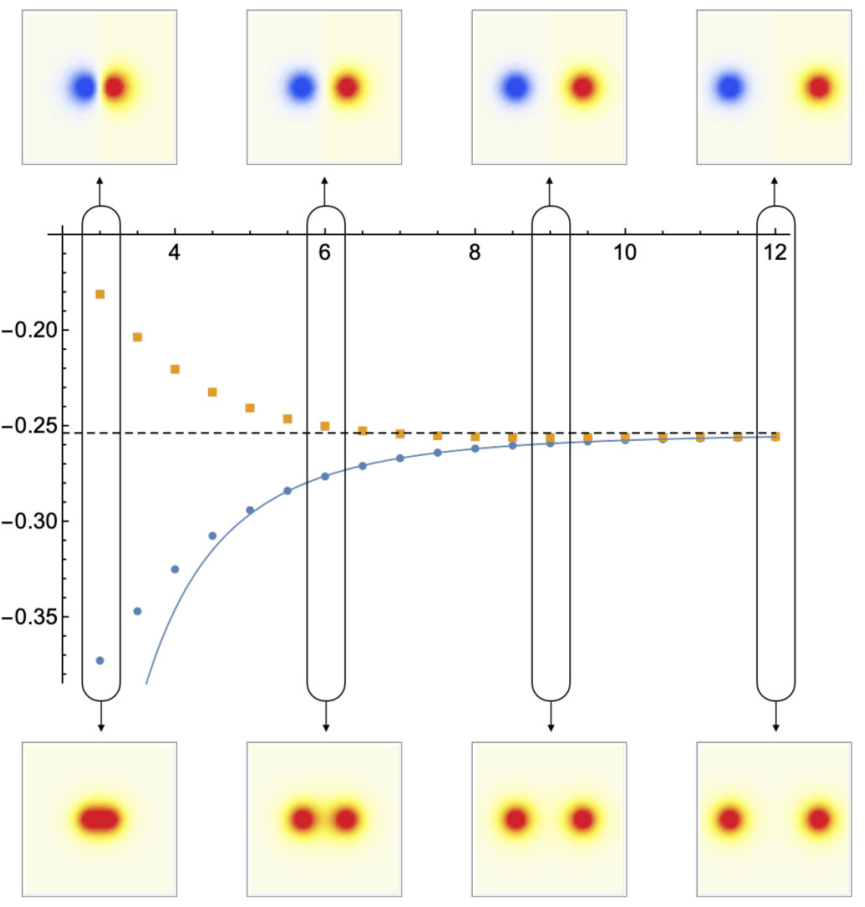

FIG. 8. The spectral flow of $1 s-1 s$ orbitals for $C_{1}=$ $2 M g_{12} \xi^{2} n_{1} N_{1} / \hbar^{2}=1$. The horizontal axis corresponds to the dimensionless distance $d / \xi$, while the vertical axis is the corresponding dimensionless energy eigenvalue $2 M N_{2} \xi^{2} \mathcal{E} / \hbar^{2}$. The dashed horizontal line shows $\mathcal{E}_{1 s}^{(1)}$, and the blue solid curve is a numerical fit shown in Eq. (22). The blue disks are for the states which are even (no nodes) for $x / \xi \rightarrow-x / \xi$, while the orange squares are for those which have odd parity. We show the wave functions of the ground states for $d / \xi=3,6,9,12$ at the bottom of the figure and those of the first excited state at the top. The spatial region of the density plots is $x / \xi \in[-10,10]$ and $y / \xi \in[-10,10]$.

of a linear node prevents the wave function from becoming shallow; instead the wave function becomes steeper in this region. Next, we consider the case of $2 M g_{12} \xi^{2} n_{1} N_{1} / \hbar^{2}=5$. As is shown in Fig. 7, each background vortex can localize the impurity not only as the ground state $(1 s)$ but also as several higher excited states $(2 p, 2 s, 3 d, 3 p, 3 s)$. In general, a wave function of an excited state has a larger length scale compared with the ground-state wave function, so covalent bonding effects start to appear for vortices placed with a larger separation compared with states with lower energy. Furthermore, when a bound state has nonzero angular momentum $(m \neq 0)$, its wave function is not axially symmetric but has directivity. As we will see in Sec. IVE, these properties of the higher excited states will give us additional contributions to the covalent bonding effect. Let us begin with the ground state and first excited state, namely, the $1 s-1 s$ mixtures. The numerical results are shown in Fig. 9. Qualitatively, there is no difference from the case with $2 M g_{12} \xi^{2} n_{1} N_{1} / \hbar^{2}=1$. The two states are almost degenerate in energy when the separation $d$ is sufficiently large. As $d$ decreases, the energy of both states decreases in a similar fashion from the asymptotic energy $\mathcal{E}_{1,0}^{(1)} \simeq-2.85$. Then, when $d$ is small enough, the spectrum undergoes splitting. The ground-state energy continuously lowers due to the $\sigma_{s-s}$ bonding, while the energy of the first excited state stops lowering and instead starts increasing due to the antibonding effect. As discussed above, the wave functions for larger $g_{12}$ are spatially smaller than those for smaller $g_{12}$. Therefore the spectral splitting occurs at relatively small $d(\lesssim 4.5 \xi)$ in comparison with the case of $C_{1}=1$.

Although we studied the impurity bound states and bonding of vortex-vortex states, the choice of the vortex charge $q$ will in fact not affect the bound states, since the impurity feels the condensate density through a potential that depends on the density of the first component [see Eq. (16)]. However, the dynamics of the coupled system would be quite different depending on the choice of $q$.

\section{E. Bonding at finite angular momentum}

Let us next look at higher excited states (2p) in which two independent states $(n, m)=(1, \pm 1)$ can coexist. Figure 10 shows the numerical results. When the vortex-vortex separation $d \gg \xi$, there are four degenerate states. Each state has directivity like a dipole, so that a total of four states can be geometrically constructed (see rightmost four panels of Fig. 10). We denote the individual $2 \mathrm{p}$ wave functions by the states $|2 p, \leftarrow\rangle,|2 p, \rightarrow\rangle,|2 p, \uparrow\rangle,|2 p, \downarrow\rangle$ expressing the direction of individual dipole axes. We consider the following products [viz., Eq. (21)] of these four states:

$$
\begin{aligned}
|0\rangle & =|2 p, \leftarrow\rangle \otimes|2 p, \rightarrow\rangle, \\
|\triangle\rangle & =|2 p, \leftarrow\rangle \otimes|2 p, \leftarrow\rangle, \\
|\square\rangle & =|2 p, \uparrow\rangle \otimes|2 p, \uparrow\rangle, \\
|\diamond\rangle & =|2 p, \uparrow\rangle \otimes|2 p, \downarrow\rangle .
\end{aligned}
$$

When the two vortices are well separated, the four states given by Eq. (23a)-(23d) are almost degenerate. However, they are not exactly degenerate as long as the distance $d$ is finite. Then, ordering these four states by their energy, we have the following: the lowest (second excited) state is denoted by |०) [Eq. (23a)] (here, the arrows indicate the direction on the bond axis; blue disks in Fig. 10) and the second-from-lowest (the third excited) state is denoted by $|\Delta\rangle$ [orange triangles,
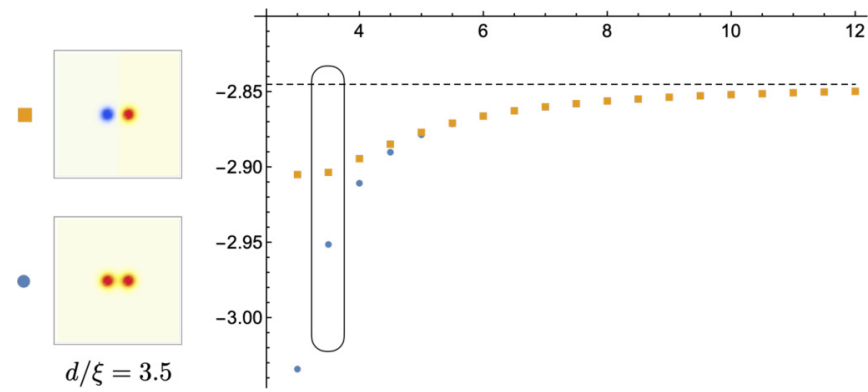

FIG. 9. The spectral flow of $1 s-1 s$ orbitals for $2 M g_{12} \xi^{2} n_{1} N_{1} / \hbar^{2}=5$. The horizontal axis corresponds to the dimensionless distance $d / \xi$, while the vertical axis is the corresponding dimensionless energy eigenvalue $2 M N_{2} \xi^{2} \mathcal{E} / \hbar^{2}$. The dashed horizontal line shows the energy of the state $\mathcal{E}_{1 s}^{(1)}$. The blue disks correspond to states which are even (no nodes) for $x / \xi \rightarrow-x / \xi$, while the orange squares are for those which have odd parity. We show the two wave functions for $d / \xi=3.5$. The spatial region of the density plots is $x / \xi \in[-10,10]$ and $y / \xi \in[-10,10]$. 


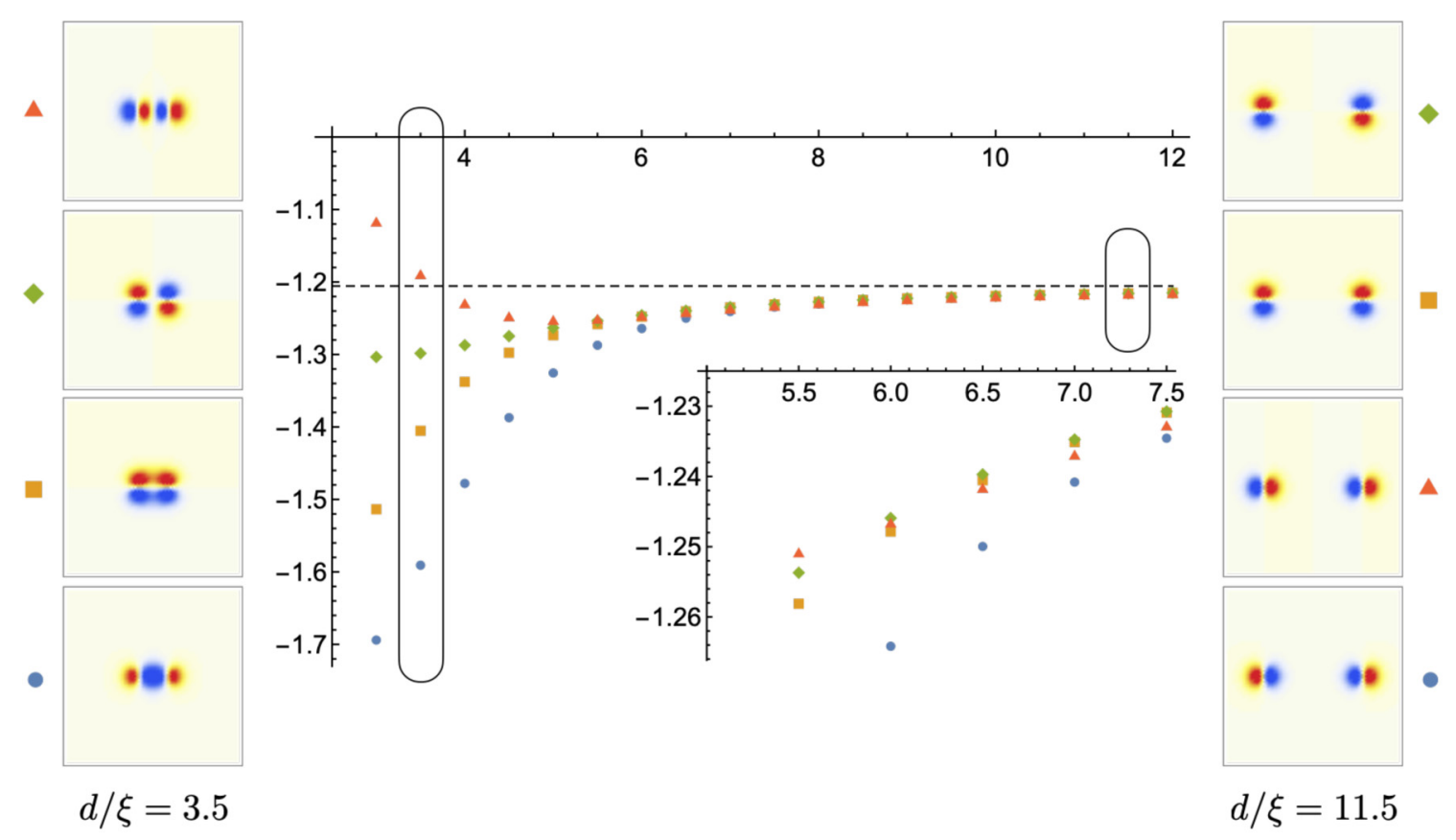

FIG. 10. The spectral flow of $2 \mathrm{p}-2 \mathrm{p}$ orbitals for $2 M g_{12} \xi^{2} n_{1} N_{1} / \hbar^{2}=5$. The horizontal axis corresponds to the dimensionless distance $d / \xi$, while the vertical axis corresponds to the dimensionless energy eigenvalue $2 M N_{2} \xi^{2} \mathcal{E} / \hbar^{2}$. The dashed horizontal line shows the energy for the state $\mathcal{E}_{2 p}^{(1)}$. The blue disks correspond to the $|0\rangle$ states [Eq. (23a)], orange triangles correspond to the $|\triangle\rangle$ states [Eq. (23b)], yellow squares correspond to the $|\square\rangle$ states [Eq. (23c)], and the $|\diamond\rangle$ states are represented as green diamonds [Eq. (23d)]. We show the wave functions for $d=11.5 \xi$ in the rightmost column and those of $d=3.5 \xi$ in the leftmost column. The spatial region of the density plots is $x / \xi \in[-10,10]$ and $y / \xi \in[-10,10]$.

Eq. (23b)], while the third-from-lowest (fourth excited state) state is denoted by $|\square\rangle$, where the arrows are orthogonal to the bond axis [yellow squares, Eq. (23c)]. The fifth excited state is denoted by $|\diamond\rangle$, where the arrows are orthogonal to the bond axis [green diamonds, Eq. (23d)].

The eigenenergies of the four states simultaneously decrease as $d$ decreases with respect to their energy, with their order unchanged. However, on the interval $6<d / \xi<6.5$, level crossings occur. Namely, the state $|\square\rangle$ becomes the third state from the fourth state, while the state $|\Delta\rangle$ is lifted from the third to fourth state (see inset of Fig. 10). The latter state $|\Delta\rangle$ is further lifted and becomes the fifth excited state as $d$ decreases. The state $|\circ\rangle$ does not participate in mixing, remaining at the lowest level. The $|0\rangle$ states' energy suddenly decreases, caused by the increasing overlap of the individual lobes of each vortex. We denote this $\sigma_{p-p}$ bonding. Following this, the remaining three states also split around $d / \xi \lesssim 5$. The energy of the second-lowest state $|\square\rangle$ also continuously decreases since two lobes of one orbital can hybridize with two lobes of the other. We denote this $\pi_{p-p}$ bonding. On the contrary, overlaps of lobes cannot occur due to their geometry for the remaining two states, $|\diamond\rangle$ and $|\Delta\rangle$. For these states, smaller displacements $d$ cause their respective energies to increase due to the antibonding effect.

Finally, we explain the higher excited states involving the $2 s$ and $3 d$ orbitals, which are shown in Fig. 11. The two states $2 s$ are denoted by

$$
\begin{aligned}
& |\circ,+\rangle=|2 s,+\rangle \otimes|2 s,+\rangle, \\
& |\triangle,-\rangle=|+, 2 s\rangle \otimes|2 s,+\rangle,
\end{aligned}
$$

and the interchanged labels appearing in the first ket on the right-hand side of Eq. (25) indicate the odd-parity version of $|2 s,+\rangle$, a general notation we adopt for the remainder of this paper. When the vortex displacement $d$ is large, the mixtures of the two $2 \mathrm{~s}$ orbitals have almost degenerate energies which asymptotically converge to $2 M N_{2} \xi^{2} \mathcal{E}_{2 s}^{(1)} / \hbar^{2} \simeq-0.46$. Since the $2 \mathrm{~s}$ orbitals are axially symmetric, the spectral flows of these almost degenerate states are qualitatively similar to the $1 s-1 s$ bound states, as explained above. However, a difference does arise from the length scales of the wave functions. Since the $2 \mathrm{~s}$ orbital is larger than the $1 \mathrm{~s}$ one, the splitting of the two degenerate states due to the $\sigma_{s-s}$ antibonding begins at relatively large $d(\lesssim 9 \xi)$ displacement for the 2 s state. However, by carefully examining the spectral flow, we find that the $\sigma_{s-s}$ bonding occurs in two stages. The first is due to overlap of the wave functions' outer shells $(d \lesssim 9 \xi)$, while the second occurs instead due to the overlap of the wave functions' inner shell $(d \lesssim 3.5 \xi)$. On the other hand, two vortices with $3 \mathrm{~d}$ orbitals have four almost degenerate states whose energies asymptotically converge to $m N_{2} \xi^{2} \mathcal{E}_{3 d}^{(1)} / \hbar^{2} \simeq-0.18$. Since the $3 \mathrm{~d}$ orbital has four lobes, we symbolically express these states as $|3 d,+\rangle$ and $|3 d, \times\rangle$. Amongst these four $3 d$ states, the lowest energy state is denoted

$$
|\square,+\rangle=|3 d,+\rangle \otimes|3 d,+\rangle,
$$

where a single lobe of one orbital facing a single lobe of another has a common color (orange squares in Fig. 11). Then, the second-lowest energy state is

$$
|\diamond,+\rangle=|3 d, \times\rangle \otimes|3 d, \times\rangle,
$$



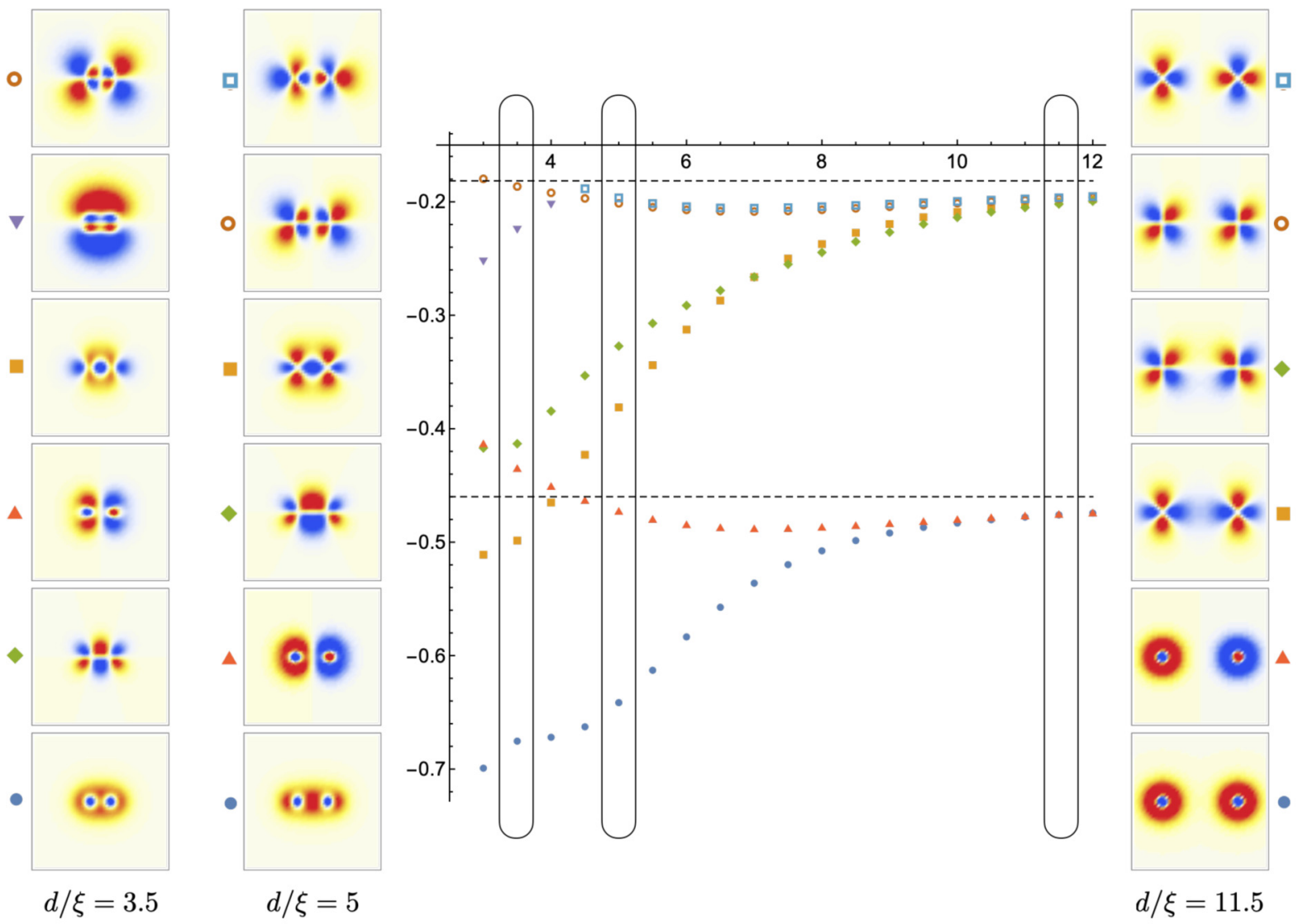

FIG. 11. The spectral flows of $2 s-2 s$ and $3 d-3 d$ orbitals (the $3 p-3 p$ mixture partially appears) for $C_{1}=5$. The horizontal axis corresponds to the dimensionless distance $d / \xi$, while the vertical axis corresponds to the dimensionless energy eigenvalue $m N_{2} \xi^{2} \mathcal{E} / \hbar^{2}$. The lower dashed horizontal line shows $m N_{2} \xi^{2} \mathcal{E}_{2 s}^{(1)} / \hbar^{2} \simeq-0.46$, and the upper one corresponds to $m N_{2} \xi^{2} \mathcal{E}_{3 d}^{(1)} / \hbar^{2} \simeq-0.18$. The blue disks correspond to |०, +$\rangle$ [Eq. (24)], red triangles denote $|\Delta,-\rangle$ states [Eq. (25)], orange squares denote $|\square,+\rangle$ [Eq. (26)], green diamonds denote $|\diamond,+\rangle[$ [Eq. (27)], open brown circles denote $|\circ,-\rangle$ [Eq. (28)], open blue squares denote $|\square,-\rangle$ [Eq. (29)], and the purple upside-down triangles denote a $\delta_{p-p}$ state. In all cases, we show the wave functions for displacements $d / \xi=3.5,5,11.5$. The spatial region of the density plots is $x / \xi \in[-10,10]$ and $y / \xi \in[-10,10]$.

where two lobes of one orbital are facing towards the two lobes of another and have a common color (green diamonds in Fig. 11). The energy eigenvalues of both of these states decrease when the vortex separation $d / \xi$ approaches (but cannot reach) zero separation. On the other hand, the odd-parity states

$$
|\circ,-\rangle=|\times, 3 d\rangle \otimes|3 d, \times\rangle
$$

correspond to open brown circles, while

$$
|\square,-\rangle=|+, 3 d\rangle \otimes|3 d,+\rangle
$$

correspond to open blue squares. The energy of the $|\circ,-\rangle$ and $|\square,-\rangle$ states are lifted by the antibonding effect in the region where $d$ is small. In the asymptotic limit $(d \gg \xi)$, the four states are almost, but not exactly degenerate. The observed ordering is $\mathcal{E}_{\square}^{+}<\mathcal{E}_{\diamond}^{+}<\mathcal{E}_{\circ}^{-}<\mathcal{E}_{\square}^{-}$. However, as $d$ decreases, level crossings occur between the $|\square,+\rangle$ and $|\diamond,+\rangle$ states. In the region $d \lesssim 5 \xi$, the lowest energy state is $|\diamond,+\rangle$. The lowering of energy is due to the $\sigma_{p-p}$ bonding for $|\square,+\rangle$, while for the $|\diamond,+\rangle$ this effect originates instead from $\pi_{d-d}$ bonding. Therefore the level crossing implies that the latter bonding is more efficient than the former one. In the region where $d$ is small enough, these states are relatively smaller than the anti- $\sigma_{s-s}$ bonded $2 s-2 s$ mixture with odd parity. We also find another type of covalent bond, $\delta_{p-p}$, which is a bound state of $3 p$ orbitals (upside-down purple triangles) appearing second from the top $(d \lesssim 4 \xi)$ in Fig. 11 . This involves bonding among four lobes, each from a $3 p$ orbital. Additionally, there are further higher excited states of $3 p-3 p$ and $3 s-3 s$ which we do not show here.

\section{v. CONCLUSIONS}

In this paper, we have modeled the interaction of a superfluid vortex with quantum impurity atoms. This has been accomplished using a two-component (binary) GrossPitaevskii formalism, where the superfluid-impurity interaction has been handled via a simple density-density interaction. The superfluid-impurity interaction has been characterized in the mass-imbalanced regime, and it was found that the presence of the impurity atoms can have a dramatic effect on the superfluid vortex, leading to distorted vortex profiles even at modest atom imbalances. The properties of vortex pairs have also been studied in the presence of impurities, and it was shown that there is an attractive splitting of the impurities' energy that depends on the signs of the vortex pairs. The splitting of the impurities' energy has been observed to be 
greater at smaller imbalances. We also investigated the timedependent dynamics of the vortex-impurity system, observing that the energies of the impurities are not significantly altered by dynamics, as long as the separation of the vortices is several healing lengths.

In the second part of this paper we considered the excited states of the coupled system. Using a Padé approximation, a model for the impurity potential generated by the vortex was constructed that allowed us to diagonalize the impurity component in the limit of small atom number imbalances, subsequently allowing the examination of the excited states of the system. In general, the number of accessible excited states depends on the details of the coupling between the two components as well as the particular choice of atom number imbalance. For deeper potentials, more excited states become accessible. The impurity orbitals of this two-dimensional system possess a two-dimensional hydrogenlike character, with higher excited states carrying finite angular momentum and being more diffuse in character as the zero (ionization) energy limit is approached. The excited impurity orbitals were then used to construct the eigenstates of a pair of like-sign vortices. The spectral flows of the impurities' lowest energy excited states revealed an attractive power-law behavior, raising the possibility of covalent bonds between vortices. The spectral flows of the excited (angular momentum carrying) states also revealed a rich behavior. Chemical bonding of pairs of vortices possessing one or two units of angular momentum display physical effects analogous to their chemical counterparts, such as the appearance of bonding and antibonding states, as well as spectral level crossings.

There are several interesting routes for future investigations. Extending this analysis to the case of fermions is a novel and worthwhile avenue, since this system is a good candidate for producing a novel matter-wave-trapped degenerate Fermi gas, analogously to the experiment of DeSalvo et al. [9]. The impurity states of this system also represent good candidates for quantum information applications, such as long-lived qubits analogous to those proposed for dark solitons $[86,87]$. Our analysis of the impurity-mediated chemical bonds could also be used as a basis to construct more elaborate forms of controllable synthetic matter, such as Toda lattices [88], vortex-impurity crystals, and impurity-associated superfluid-insulator transitions. Furthermore, collisions between multiple vortex carrying impurities would allow for the simulation of synthetic chemical reactions. Our work also represents a tool in the growing field of atomtronics [89]; the ability to construct synthetic chemical bonds yields a useful paradigm in this emerging discipline.

\section{ACKNOWLEDGMENTS}

This work is supported in part by Japan Society of Promotion of Science (JSPS) Grant-in-Aid for Scientific Research [KAKENHI Grants No. JP20K14376 (M.E.), No. JP19K03839 (M.E.), and JP18H01217 (M.N.)] and also by MEXT KAKENHI Grant-in-Aid for Scientific Research on Innovative Areas, Discrete Geometric Analysis for Materials Design, No. JP17H06462 (M.E.) from the MEXT of Japan.

\section{APPENDIX: VARIATIONAL INTEGRALS}

In this Appendix we list the integrals required to evaluate the various contributions to the variational energy, Eqs. (6a)(6d) in Sec. III B of the main text. Below we explain the origin of the individual integrals:

$$
\begin{gathered}
\int_{0}^{\infty} d u u\left[\frac{1}{\beta^{2}(\beta+1)^{2}}-\left(\frac{1}{\beta+\exp \left(-u^{2}\right)}-\frac{1}{\beta+1}\right)^{2}\right]=\frac{(\beta-1) \ln \left(\frac{\beta}{\beta+1}\right)+1}{2 \beta^{2}(\beta+1)}, \\
\int_{0}^{\infty} d u u\left[\frac{\partial}{\partial u} \frac{1}{\beta+\exp \left(-u^{2}\right)}\right]^{2}=\frac{1}{3 \beta^{2}}\left[\ln \left(\frac{\beta+1}{\beta}\right)-\frac{\beta}{(\beta+1)^{2}}\right], \\
\int_{0}^{\infty} d u u\left[\frac{1}{\beta^{2}(\beta+1)^{2}}-\left(\frac{1}{\beta+\exp \left(-u^{2}\right)}-\frac{1}{\beta+1}\right)^{2}\right]^{2}=\frac{6(\beta+1)(\beta-1)^{2} \ln \left(\frac{\beta+1}{\beta}\right)+1+3 \beta(3-2 \beta)}{12 \beta^{4}(\beta+1)^{3}} .
\end{gathered}
$$

To calculate the normalization of the variational function $\psi_{1}^{\mathrm{var}}(x, y)$, the first integral, given by Eq. (A1), is used. To evaluate the contribution from the density of the variational kinetic energy [Eq. (3a)] for the vortex, the second integral, given by Eq. (A2), is required. Finally, Eq. (A3) is needed to calculate the van der Waals contribution arising from Eq. (3b).
[1] F. Chevy, K. W. Madison, and J. Dalibard, Measurement of the Angular Momentum of a Rotating Bose-Einstein Condensate, Phys. Rev. Lett. 85, 2223 (2000).

[2] C. Raman, J. R. Abo-Shaeer, J. M. Vogels, K. Xu, and W. Ketterle, Vortex Nucleation in a Stirred Bose-Einstein Condensate, Phys. Rev. Lett. 87, 210402 (2001).

[3] J. R. Abo-Shaeer, C. Raman, J. M. Vogels, and W. Ketterle, Observation of vortex lattices in Bose-Einstein condensates, Science 292, 476 (2001).
[4] A. L. Fetter, Rotating trapped Bose-Einstein condensates, Rev. Mod. Phys. 81, 647 (2009).

[5] F. Dalfovo, S. Giorgini, L. P. Pitaevskii, and S. Stringari, Theory of Bose-Einstein condensation in trapped gases, Rev. Mod. Phys. 71, 463 (1999).

[6] N. B. Jørgensen, L. Wacker, K. T. Skalmstang, M. M. Parish, J. Levinsen, R. S. Christensen, G. M. Bruun, and J. J. Arlt, Observation of Attractive and Repulsive Polarons in a BoseEinstein Condensate, Phys. Rev. Lett. 117, 055302 (2016). 
[7] M.-G. Hu, M. J. V. de Graaff, D. Kedar, J. P. Corson, E. A. Cornell, and D. S. Jin, Bose Polarons in the Strongly Interacting Regime, Phys. Rev. Lett. 117, 055301 (2016).

[8] A. Schirotzek, C.-H. Wu, A. Sommer, and M. W. Zwierlein, Observation of Fermi Polarons in a Tunable Fermi Liquid of Ultracold Atoms, Phys. Rev. Lett. 102, 230402 (2009).

[9] B. J. DeSalvo, K. Patel, J. Johansen, and C. Chin, Observation of a Degenerate Fermi Gas Trapped by a Bose-Einstein Condensate, Phys. Rev. Lett. 119, 233401 (2017).

[10] R. M. Kalas and D. Blume, Interaction-induced localization of an impurity in a trapped Bose-Einstein condensate, Phys. Rev. A 73, 043608 (2006).

[11] K. Sacha and E. Timmermans, Self-localized impurities embedded in a one-dimensional Bose-Einstein condensate and their quantum fluctuations, Phys. Rev. A 73, 063604 (2006).

[12] M. J. Edmonds, J. Helm, and T. Busch, Coherent impurity transport in an attractive binary Bose-Einstein condensate, New J. Phys. 21, 053019 (2019).

[13] T. P. Meyrath, F. Schreck, J. L. Hanssen, C. S. Chuu, and M. G. Raizen, Bose-Einstein condensate in a box, Phys. Rev. A 71, 041604(R) (2005).

[14] J. J. P. van Es, P. Wicke, A. H. van Amerongen, C. Rétif, S. Whitlock, and N. J. van Druten, Box traps on an atom chip for one-dimensional quantum gases, J. Phys. B: At. Mol. Opt. Phys. 43, 155002 (2010).

[15] R. Saint-Jalm, P. C. M. Castilho, É. Le Cerf, B. BakkaliHassani, J.-L. Ville, S. Nascimbene, J. Beugnon, and J. Dalibard, Dynamical Symmetry and Breathers in a TwoDimensional Bose Gas, Phys. Rev. X 9, 021035 (2019).

[16] A. L. Gaunt, T. F. Schmidutz, I. Gotlibovych, R. P. Smith, and Z. Hadzibabic, Bose-Einstein Condensation of Atoms in a Uniform Potential, Phys. Rev. Lett. 110, 200406 (2013).

[17] N. Navon, A. L. Gaunt, R. P. Smith, and Z. Hadzibabic, Emergence of a turbulent cascade in a quantum gas, Nature (London) 539, 72 (2016).

[18] K. Hueck, N. Luick, L. Sobirey, J. Siegl, T. Lompe, and H. Moritz, Two-Dimensional Homogeneous Fermi Gases, Phys. Rev. Lett. 120, 060402 (2018).

[19] G. W. Stagg, N. G. Parker, and C. F. Barenghi, Quantum analogues of classical wakes in Bose-Einstein condensates, J. Phys. B: At. Mol. Opt. Phys. 47, 095304 (2014).

[20] L. Chomaz, L. Corman, T. Bienaimé, R. Desbuquois, C. Weitenberg, S. Nascimbène, J. Beugnon, and J. Dalibard, Emergence of coherence via transverse condensation in a uniform quasi-two-dimensional Bose gas, Nat. Commun. 6, 6162 (2015).

[21] S. K. Adhikari, Vortex-lattice in a uniform Bose-Einstein condensate in a box trap, J. Phys.: Condens. Matter 31, 275401 (2019).

[22] K. Kasamatsu, M. Tsubota, and M. Ueda, Vortex Phase Diagram in Rotating Two-Component Bose-Einstein Condensates, Phys. Rev. Lett. 91, 150406 (2003).

[23] L. Mingarelli and R. Barnett, Exotic Vortex Lattices in Binary Repulsive Superfluids, Phys. Rev. Lett. 122, 045301 (2019).

[24] M. Edmonds and M. Nitta, Vortex patterns of atomic BoseEinstein condensates in a density-dependent gauge potential, Phys. Rev. A 102, 011303(R) (2020).

[25] J. E. H. Braz and H. Terças, Bound-state spectrum of an impurity in a quantum vortex, Phys. Rev. A 101, 023607 (2020).
[26] R. Clark, Self-trapped electrons in liquid helium II, Phys. Lett. 16, 42 (1965).

[27] A. Villois and H. Salman, Vortex nucleation limited mobility of free electron bubbles in the Gross-Pitaevskii model of a superfluid, Phys. Rev. B 97, 094507 (2018).

[28] C. Barenghi and N. G. Parker, A Primer on Quantum Fluids (Springer, Berlin, 2016).

[29] M. C. Tsatsos, P. E. S. Tavares, A. Cidrim, A. R. Fritsch, M. A. Caracanhas, F. E. A. dos Santos, C. F. Barenghi, and V. S. Bagnato, Quantum turbulence in trapped atomic Bose-Einstein condensates, Phys. Rep. 622, 1 (2016).

[30] A. C. White, B. P. Anderson, and V. S. Bagnato, Vortices and turbulence in trapped atomic condensates, Proc. Natl. Acad. Sci. USA 111, 4719 (2014).

[31] W. J. Kwon, J. H. Kim, S. W. Seo, and Y. Shin, Observation of von Kármán Vortex Street in an Atomic Superfluid Gas, Phys. Rev. Lett. 117, 245301 (2016).

[32] I.-K. Liu, S. Donadello, G. Lamporesi, G. Ferrari, S.-C. Gou, F. Dalfovo, and N. P. Proukakis, Dynamical equilibration across a quenched phase transition in a trapped quantum gas, Commun. Phys. 1, 24 (2018)

[33] T. W. Neely, E. C. Samson, A. S. Bradley, M. J. Davis, and B. P. Anderson, Observation of Vortex Dipoles in an Oblate BoseEinstein Condensate, Phys. Rev. Lett. 104, 160401 (2010).

[34] M. M. Cawte, X. Yu, B. P. Anderson, and A. S. Bradley, Snell's Law for a vortex dipole in a Bose-Einstein condensate, SciPost Phys. 6, 032 (2019).

[35] G. Yang, S. Zhang, and J. Jin, Collision of parallel vortex dipoles in a Bose-Einstein condensate, J. Phys. B: At. Mol. Opt Phys. 52, 065201 (2019).

[36] S. Donadello, S. Serafini, M. Tylutki, L. P. Pitaevskii, F Dalfovo, G. Lamporesi, and G. Ferrari, Observation of Solitonic Vortices in Bose-Einstein Condensates, Phys. Rev. Lett. 113 065302 (2014).

[37] Y. Castin and R. Dum, Bose-Einstein condensates with vortices in rotating traps, Eur. Phys. J. D 7, 399 (1999).

[38] M. Tsubota, K. Kasamatsu, and M. Ueda, Vortex lattice formation in a rotating Bose-Einstein condensate, Phys. Rev. A 65, 023603 (2002).

[39] M. Cozzini and S. Stringari, Macroscopic dynamics of a BoseEinstein condensate containing a vortex lattice, Phys. Rev. A 67, 041602(R) (2003).

[40] L. J. O'Riordan, A. C. White, and T. Busch, Moiré superlattice structures in kicked Bose-Einstein condensates, Phys. Rev. A 93, 023609 (2016).

[41] L. J. O'Riordan and T. Busch, Topological defect dynamics of vortex lattices in Bose-Einstein condensates, Phys. Rev. A 94 053603 (2016).

[42] M. Lewenstein, A. Sanpera, and V. Ahufinger, Ultracold Atoms in Optical Lattices: Simulating Quantum Many-Body Systems (Oxford University Press, Oxford, 2012).

[43] D. T. Son and M. A. Stephanov, Domain walls of relative phase in two-component Bose-Einstein condensates, Phys. Rev. A 65 063621 (2002).

[44] K. Kasamatsu, M. Tsubota, and M. Ueda, Vortex Molecules in Coherently Coupled Two-Component Bose-Einstein Condensates, Phys. Rev. Lett. 93, 250406 (2004).

[45] M. Eto and M. Nitta, Vortex trimer in three-component BoseEinstein condensates, Phys. Rev. A 85, 053645 (2012). 
[46] M. Eto and M. Nitta, Vortex graphs as N-omers and $\mathbb{C} P^{N-1}$ skyrmions in N-component Bose-Einstein condensates, EPL 103, 60006 (2013).

[47] M. Cipriani and M. Nitta, Crossover between Integer and Fractional Vortex Lattices in Coherently Coupled Two-Component Bose-Einstein Condensates, Phys. Rev. Lett. 111, 170401 (2013).

[48] M. Tylutki, L. P. Pitaevskii, A. Recati, and S. Stringari, Confinement and precession of vortex pairs in coherently coupled Bose-Einstein condensates, Phys. Rev. A 93, 043623 (2016).

[49] M. Eto and M. Nitta, Confinement of half-quantized vortices in coherently coupled Bose-Einstein condensates: Simulating quark confinement in a QCD-like theory, Phys. Rev. A 97, 023613 (2018).

[50] M. Eto, K. Ikeno, and M. Nitta, Collision dynamics and reactions of fractional vortex molecules in coherently coupled Bose-Einstein condensates, Phys. Rev. Research 2, 033373 (2020).

[51] M. Kobayashi, M. Eto, and M. Nitta, Berezinskii-KosterlitzThouless Transition of Two-Component Bose Mixtures with Intercomponent Josephson Coupling, Phys. Rev. Lett. 123, 075303 (2019).

[52] A. E. Leanhardt, A. Görlitz, A. P. Chikkatur, D. Kielpinski, Y. Shin, D. E. Pritchard, and W. Ketterle, Imprinting Vortices in a Bose-Einstein Condensate using Topological Phases, Phys. Rev. Lett. 89, 190403 (2002).

[53] T. Mizushima, K. Machida, and T. Kita, Mermin-Ho Vortex in Ferromagnetic Spinor Bose-Einstein Condensates, Phys. Rev. Lett. 89, 030401 (2002).

[54] J. P. Martikainen, A. Collin, and K. A. Suominen, Coreless vortex ground state of the rotating spinor condensate, Phys. Rev. A 66, 053604 (2002).

[55] S. Mukerjee, C. Xu, and J. E. Moore, Topological Defects and the Superfluid Transition of the $s=1$ Spinor Condensate in Two Dimensions, Phys. Rev. Lett. 97, 120406 (2006).

[56] U. Leonhardt and G. E. Volovik, How to create an Alice string (half-quantum vortex) in a vector Bose-Einstein condensate, $\mathrm{J}$ Exp. Theor. Phys. 72, 46 (2000).

[57] T. Ohmi and K. Machida, Bose-Einstein condensation with internal degrees of freedom in alkali atom gases, J. Phys. Soc. Jpn. 67, 1822 (1998).

[58] S. Kobayashi, Y. Kawaguchi, M. Nitta, and M. Ueda, Topological classification of vortex-core structures of spin-1 BoseEinstein condensates, Phys. Rev. A 86, 023612 (2012).

[59] J. Lovegrove, M. O. Borgh, and J. Ruostekoski, Energetic Stability of Coreless Vortices in Spin-1 Bose-Einstein Condensates with Conserved Magnetization, Phys. Rev. Lett. 112, 075301 (2014).

[60] M. O. Borgh, M. Nitta, and J. Ruostekoski, Stable Core Symmetries and Confined Textures for a Vortex Line in a Spinor Bose-Einstein Condensate, Phys. Rev. Lett. 116, 085301 (2016).

[61] Y. Kawaguchi and M. Ueda, Spinor Bose-Einstein condensates, Phys. Rep. 520, 253 (2012).

[62] K. Kasamatsu, M. Tsubota, and M. Ueda, Vortices in multicomponent Bose-Einstein condensates, Int. J. Mod. Phys. B 19, 1835 (2005).

[63] M. Eto, K. Kasamatsu, M. Nitta, H. Takeuchi, and M. Tsubota, Interaction of half-quantized vortices in two-component BoseEinstein condensates, Phys. Rev. A 83, 063603 (2011).
[64] K. Kasamatsu, M. Eto, and M. Nitta, Short-range intervortex interaction and interacting dynamics of half-quantized vortices in two-component Bose-Einstein condensates, Phys. Rev. A 93, 013615 (2016).

[65] E. J. Mueller and T.-L. Ho, Two-Component Bose-Einstein Condensates with a Large Number of Vortices, Phys. Rev. Lett. 88, 180403 (2002).

[66] W. C. Yang, C. Y. Xia, M. Nitta, and H. B. Zeng, Fractional and integer vortex dynamics in strongly coupled two-component Bose-Einstein condensates from AdS/CFT correspondence, Phys. Rev. D 102, 046012 (2020).

[67] H. T. C. Stoof, E. Vliegen, and U. Al Khawaja, Monopoles in an Antiferromagnetic Bose-Einstein Condensate, Phys. Rev. Lett. 87, 120407 (2001)

[68] J. Ruostekoski and J. R. Anglin, Monopole Core Instability and Alice Rings in Spinor Bose-Einstein Condensates, Phys. Rev. Lett. 91, 190402 (2003).

[69] M. W. Ray, E. Ruokokoski, S. Kandel, M. Möttönen, and D. S. Hall, Observation of Dirac monopoles in a synthetic magnetic field, Nature (London) 505, 657 (2014).

[70] J. Y. Choi, W. J. Kwon, and Y. Shin, Observation of Topologically Stable 2D Skyrmions in an Antiferromagnetic Spinor Bose-Einstein Condensate, Phys. Rev. Lett. 108, 035301 (2012).

[71] U. A. Khawaja and H. T. C. Stoof, Skyrmion physics in Bose-Einstein ferromagnets, Phys. Rev. A 64, 043612 (2001).

[72] J. Ruostekoski and J. R. Anglin, Creating Vortex Rings and Three-Dimensional Skyrmions in Bose-Einstein Condensates, Phys. Rev. Lett. 86, 3934 (2001).

[73] R. A. Battye, N. R. Cooper, and P. M. Sutcliffe, Stable Skyrmions in Two-Component Bose-Einstein Condensates, Phys. Rev. Lett. 88, 080401 (2002).

[74] M. Nitta, K. Kasamatsu, M. Tsubota, and H. Takeuchi, Creating vortons and three-dimensional skyrmions from domain-wall annihilation with stretched vortices in Bose-Einstein condensates, Phys. Rev. A 85, 053639 (2012).

[75] T. Kawakami, T. Mizushima, M. Nitta, and K. Machida, Stable Skyrmions in $S U$ (2) Gauged Bose-Einstein Condensates, Phys. Rev. Lett. 109, 015301 (2012).

[76] Y. Kawaguchi, M. Nitta, and M. Ueda, Knots in a Spinor Bose-Einstein Condensate, Phys. Rev. Lett. 100, 180403 (2008).

[77] D. S. Hall, M. W. Ray, K. Tiurev, E. Ruokokoski, A. H. Gheorghe, and M. Möttönen, Tying quantum knots, Nat. Phys. 12, 478 (2016).

[78] T. Ollikainen, A. Blinova, M. Möttönen, and D. S. Hall, Decay of a Quantum Knot, Phys. Rev. Lett. 123, 163003 (2019).

[79] K. Kasamatsu, H. Takeuchi, M. Nitta, and M. Tsubota, Analogues of D-branes in Bose-Einstein condensates, J. High Energy Phys. 11 (2010) 068.

[80] K. Kasamatsu, H. Takeuchi, and M. Nitta, D-brane solitons and boojums in field theory and Bose-Einstein condensates, J. Phys.: Condens. Matter 25, 404213 (2013).

[81] K. Kasamatsu, H. Takeuchi, M. Tsubota, and M. Nitta, Wallvortex composite solitons in two-component Bose-Einstein condensates, Phys. Rev. A 88, 013620 (2013).

[82] L. Rutherford, J. Goold, T. Busch, and J. F. McCann, Transport, atom blockade, and output coupling in a Tonks-Girardeau gas, Phys. Rev. A 83, 055601 (2011). 
[83] S. Palzer, C. Zipkes, C. Sias, and M. Köhl, Quantum Transport through a Tonks-Girardeau Gas, Phys. Rev. Lett. 103, 150601 (2009).

[84] C. J. Pethick and H. Smith, Bose-Einstein Condensation in Dilute Gases (Cambridge University Press, Cambridge, 2002).

[85] C. Rorai, K. R. Sreenivasan, and M. E. Fisher, Propagating and annihilating vortex dipoles in the Gross-Pitaevskii equation, Phys. Rev. B 88, 134522 (2013).

[86] M. I. Shaukat, E. V. Castro, and H. Terças, Quantum dark solitons as qubits in Bose-Einstein condensates, Phys. Rev. A 95, 053618 (2017).
[87] M. I. Shaukat, E. V. Castro, and H. Terças, Entanglement sudden death and revival in quantum dark-soliton qubits, Phys. Rev. A 98, 022319 (2018).

[88] M. Toda, Vibration of a chain with nonlinear interaction, J. Phys. Soc. Jpn. 22, 431 (1967).

[89] L. Amico, M. Boshier, G. Birkl, A. Minguzzi, C. Miniatura, L.-C. Kwek, D. Aghamalyan, V. Ahufinger, D. Anderson, N. Andrei, A. S. Arnold, M. Baker, T. A. Bell, T. Bland, J. P. Brantut, D. Cassettari, W. J. Chetcuti, F. Chevy, R. Citro, S. De Palo et al., State of the art and perspective on Atomtronics, arXiv:2008.04439. 